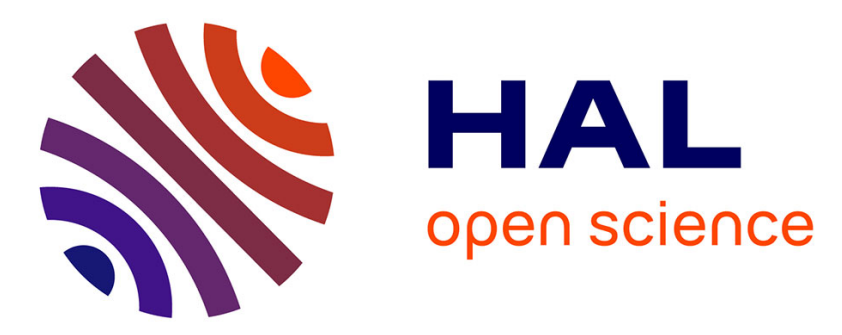

\title{
A Kalman filter adapted to the estimation of mean gradients in the large-eddy simulation of unsteady turbulent flows
}

Jérôme Boudet, Emmanuel Lévêque, Pierre Borgnat, Adrien Cahuzac, Marc C. Jacob

\section{To cite this version:}

Jérôme Boudet, Emmanuel Lévêque, Pierre Borgnat, Adrien Cahuzac, Marc C. Jacob. A Kalman filter adapted to the estimation of mean gradients in the large-eddy simulation of unsteady turbulent flows. Computers and Fluids, 2016, 127, pp.65-77. 10.1016/j.compfluid.2015.12.006 . hal-01393342

\section{HAL Id: hal-01393342 \\ https://hal.science/hal-01393342}

Submitted on 31 Jul 2019

HAL is a multi-disciplinary open access archive for the deposit and dissemination of scientific research documents, whether they are published or not. The documents may come from teaching and research institutions in France or abroad, or from public or private research centers.
L'archive ouverte pluridisciplinaire HAL, est destinée au dépôt et à la diffusion de documents scientifiques de niveau recherche, publiés ou non, émanant des établissements d'enseignement et de recherche français ou étrangers, des laboratoires publics ou privés. 


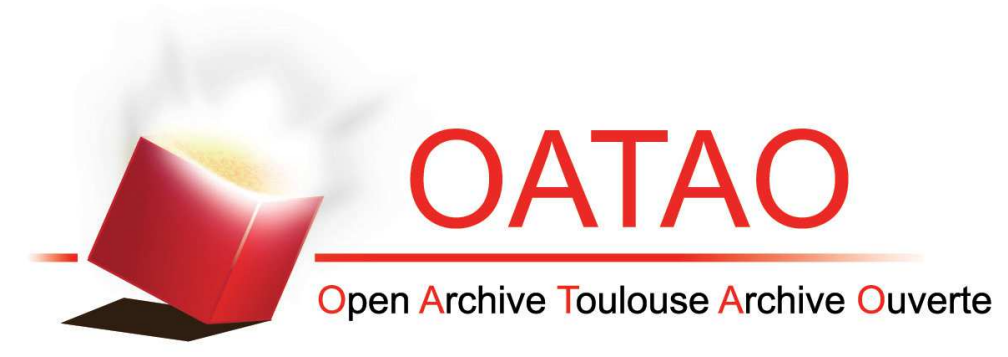

\section{Open Archive Toulouse Archive Ouverte (OATAO)}

OATAO is an open access repository that collects the work of some Toulouse researchers and makes it freely available over the web where possible.

This is an author's version published in: https://oatao.univ-toulouse.fr/24073

Official URL : https://doi.org/10.1016/j.compfluid.2015.12.006

\section{To cite this version :}

Boudet, Jérôme and Lévêque, Emmanuel and Borgnat, Pierre and Cahuzac, Adrien and Jacob, Marc C. A Kalman filter adapted to the estimation of mean gradients in the large-eddy simulation of unsteady turbulent flows. (2016) Computers and Fluids, 127. 65-77. ISSN 0045-7930

Any correspondence concerning this service should be sent to the repository administrator: tech-oatao@listes-diff.inp-toulouse.fr 


\title{
A Kalman filter adapted to the estimation of mean gradients in the large-eddy simulation of unsteady turbulent flows
}

\author{
J. Boudet ${ }^{a}$, E. Lévêque ${ }^{\mathrm{a}, *}$, P. Borgnat ${ }^{\mathrm{b}}$, A. Cahuzac ${ }^{\mathrm{a}}$, M.C. Jacob $^{\mathrm{a}}$ \\ a LMFA, Ecole Centrale de Lyon, CNRS, Université de Lyon, 36 avenue Guy de Collongue, F-69134 Ecully Cedex, France \\ ${ }^{\mathrm{b}}$ Laboratoire de Physique de l'École normale supérieure de Lyon, CNRS, Université de Lyon, 46 allée d'Italie, F-69364 Lyon Cedex 7, France
}

\section{A R T I C L E I N F O}

\section{Keywords:}

Large-eddy simulation

Unsteady turbulent flows

Kalman filter

\begin{abstract}
A B S T R A C T
A computationally-efficient method based on Kalman filtering is introduced to capture "on the fly" the lowfrequency (or very large-scale) patterns of a turbulent flow in a large-eddy simulation (LES). This method may be viewed as an adaptive exponential smoothing in time with a varying cut-off frequency that adjusts itself automatically to the local rate of turbulence of the simulated flow. It formulates as a recursive algorithm, which requires only few arithmetic operations per time step and has very low memory usage. In practice, this smoothing algorithm is used in LES to evaluate the low-frequency component of the rate of strain, and implement a shear-improved variant of the Smagrosinky's subgrid-scale viscosity. Such approach is primarily devoted to the simulation of turbulent flows that develop large-scale unsteadiness associated with strong shear variations. As a severe test case, the flow past a circular cylinder at Reynolds number $\operatorname{Re}_{D}=4.7 \times 10^{4}$ (in the subcritical turbulent regime) is examined in details. Aerodynamic and aeroacoustic features including spectral analysis of the velocity and the far-field pressure are found in good agreement with various experimental data. The Kalman filter suitably captures the pulsating behavior of the flow and provides meaningful information about the large-scale dynamics. Finally, the robustness of the method is assessed by varying the parameters entering in the calibration of the Kalman filter.
\end{abstract}

\section{Motivations}

The numerical simulation of turbulent flows in geometries of engineering interest can be accomplished with various levels of approximation, yielding a more or less detailed representation of the flow. The so-called direct simulation, in which the equations of motion are discretized and solved directly, is obviously the most straightforward approach. If the mesh is sufficiently fine to resolve even the smallest eddies, and if the numerical scheme limits dispersion and dissipation errors, this method yields an accurate time-dependent representation of the flow [1]. Unfortunately, its applicability is limited to simple geometries at relatively low Reynolds numbers. The reason is twofold. First, the drawback of using highly accurate schemes is unavoidably a lack of flexibility to handle complex geometries and general boundary conditions. Second, the resolution of turbulent fluid motions at high Reynolds numbers requires a prohibitive number of grid points, especially in near-wall regions where thin vortical structures develop [2]. Therefore, in practical situations, the direct approach is often abandoned in favor of approximate, but numerically tractable, computations.

\footnotetext{
* Corresponding author. Tel.: +33 472186168.

E-mail address: emmanuel.leveque@ec-lyon.fr (E. Lévêque).
}

In a large-eddy simulation, usually referred to as LES in the literature, the grid resolution is deliberately reduced so that only the largescale motions of the fluid are captured numerically. This is physically justifiable since the large-sized eddies contain most of the kinetic energy of the flow, and their strengths make them the efficient carriers of mass, momentum, heat, etc. On the contrary, small-sized eddies are mainly responsible for dissipation and contribute little to transport and mixing. The large-scale dynamics is solution of the original flow equations, e.g. the Navier-Stokes equations, supplemented by an unknown term accounting for the stress exerted by the unresolved subgrid-scale motions on the simulated flow. A common thread is to assume that this stress is essentially responsible for a diffusive transport of fluid momentum at grid scale, which in turn calls for the modeling of a subgrid-scale viscosity [3]. This viscosity depends on space and time, and is related to the (subgrid-scale) turbulent dynamics.

In the context of engineering flows, which may experience strong unsteady events such as boundary-layer separation, vortex shedding or disturbances induced by a moving body, e.g. a turbine blade, the modeling of the subgrid-scale viscosity is recognized to be a difficult problem. Strong unsteadiness generally occurs at low frequencies in comparison with turbulent fluctuations in the bulk and is often associated with large amplitudes of the rate of strain (or shear). In this respect, a refinement of the Smagorinsky's model [4] has been 
proposed recently. Namely, the so-called shear-improved Smagorinsky's model (SISM) [5] accounts explicitly for the mean part (in the sense of statistical average) of the rate of strain to correct the Smagorinsky's viscosity. The resulting viscosity expresses as

$v_{\text {sgs }}(\mathbf{x}, t)=\left(C_{s} \Delta(\mathbf{x})\right)^{2}(|\bar{S}(\mathbf{x}, t)|-\mathcal{S}(\mathbf{x}, t))$,

where $C_{s}=0.18$ is the standard Smagorinsky constant [6], $\Delta(\mathbf{x})$ is the local grid spacing (at position $\mathbf{x}$ ) and $|\overline{\mathrm{S}}(\mathbf{x}, t)|$ is the norm of the resolved rate-of-strain tensor: $|\overline{\mathrm{S}}|=\sqrt{2 \sum_{i j} \overline{\mathrm{S}}_{i j} \overline{\mathrm{S}}_{i j}}$. In the notation, the overline recalls that the flow quantity is discretized at a grid resolution that may be coarse compared to the size of the smallest turbulent eddies. In Eq. (1), the correcting term to the Smagorinsky's viscosity is $\mathcal{S}(\mathbf{x}, t)=\mid \bar{S} x, t) \mid$, where the tilde refers typically to a lowpass filtering (as discussed below). Interestingly, the SISM does not call for any adjustable parameter besides $C_{s}=0.18$, which is fixed for all flows. There is no need for an ad-hoc damping function nor any kind of dynamic adjustment in near-wall regions [7]. The simplicity and manageability of the original Smagorinsky's model are therefore preserved.

The theoretical basis of the SISM was put forward on account of numerical and experimental studies on shear effects in nonhomogeneous turbulence [9].

In the context of subgrid-scale modeling, it shares obvious similarities with the model originally introduced by Schumann in 1975 , which relies on a two-part eddy-viscosity accounting for the interplay between the nonlinear energy cascade present in isotropic turbulence and mean-shear effects associated with anisotropy [10]. However, the SISM clearly differs from Schumann's proposal. This later requires an empirical prescription for the "inhomogeneous eddy-viscosity", whereas the subgrid-scale viscosity is explicit in the SISM and arises naturally from the scale-by-scale energy budget established from the Navier-Stokes equations [5]. Another important point is that the SISM cannot be obtained by just simplifying Schumann's formulation. Let us note that variants of Schumann's model have also been proposed by Moin and Kim in 1982 [11] and followed by Horuiti in 1987 [12], and one can add the anisotropic version introduced by Sullivan et al. in 1994 [13].

Including anisotropy effects in the SGS modeling has been addressed in many different ways. The SISM relies on a decomposition of the resolved flow into a statistically-averaged part and a fluctuating part. An alternative decomposition into a large-scale and a smallscale component has been extensively explored. This refers for instance to the variational multi-scale (VMS) method, which originates with the works of Temam on multi-level methods [14] and has been developed by Hughes et al. [15] and many others thereafter (see [16] for a review). This decomposition arises from the motivation to build an eddy-viscosity on either the small-scale or the large-scale part of the velocity field, and make it act on the small-scale part of the resolved motions only. One can also mention the filtered structurefunction model introduced by Ducros et al. [17] that consists of removing the large-scale fluctuations of the velocity field before computing its second-order structure function.

An important requirement of the SISM is to evaluate the mean component of the rate of strain (in the sense of ensemble average) as the simulation progresses. In practice, ensemble average may be approximated by space average over directions of homogeneity, whenever it is possible, e.g. in a plane-channel flow. When it is not, time average may be used instead if the flow is statistically time-invariant. However, many engineering flows do not allow such approximations and an alternative estimation must be found, which is the issue addressed in the present work. Our proposal is to assume that the mean flow may be approximated by the low-frequency component of the velocity field, including a possible (quasi-)deterministic unsteadiness, and that the turbulent component adds itself to this "unsteady" mean (see [8] for more details). An original method based on Kalman filtering in the time domain is investigated. Importantly, this filter corrects its cut-off frequency automatically according to the local turbulent rate of the flow. It is therefore well-adapted to strongly inhomogeneous and unsteady flows. This method is fully local in space and applies independently at each grid point. It is thus convenient to treat complex-geometry flows, possibly integrated on unstructured grids. The physical fundamentals of this method have already been presented in a companion paper [8], the focus is here on computational and validation aspects, including a spectral analysis.

\section{Kalman filtering adapted to turbulent flows}

\subsection{Exponential smoothing as baseline method}

A simple way to extract the low-frequency component of a digital signal is to apply a weighted moving average (in time) to this signal. In the context of computational fluid dynamics, this moving average should be applied at each grid point and every time step, making the cost of this operation highly selective. The simplest solution is certainly to consider an exponentially-weighted moving average, or exponential smoothing $[18,19]$. See [20-22] for existing applications of exponential smoothing in the context of LES. The main advantage of the exponential smoothing is that it can be formulated in a very convenient recursive manner:

$\widetilde{\mathbf{u}}^{(n+1)}=(1-\alpha) \cdot \widetilde{\mathbf{u}}^{(n)}+\alpha \cdot \overline{\mathbf{u}}^{(n+1)}$,

where $\widetilde{\mathbf{u}}^{(n)}$ denotes the smoothed velocity (at time $n$ ) whereas $\overline{\mathbf{u}}^{(n)}$ is the instantaneous velocity. The smoothing factor $0<\alpha<1$ controls the weights of the past observations in the average (a higher $\alpha$ discounts older observations faster). The exponential smoothing is formally equivalent to a first-order low-pass filter with a cut-off frequency $f_{c}$ related to the smoothing factor by

$\alpha \simeq \frac{2 \pi f_{c} \Delta t}{\sqrt{3}} \approx 3.628 f_{c} \cdot \Delta t$

where $\Delta t$ is the time step of the velocity signal (see [8] for a proof).

In the exponential smoothing, the key point is to update at each time step the smoothed quantity (here the velocity) by taking into account the new data point. It is computationally efficient since it requires only the storage of the (previous) smoothed quantity. Also, the initialization of the algorithm is very simple: $\widetilde{\mathbf{u}}^{(0)}=\overline{\mathbf{u}}^{(0)}$. In the context of complex turbulent flows, an obvious limitation of this method is to select a unique physically-relevant cut-off frequency for the whole flow. In practice, the smoothing factor is expected to vary in space and time according to the large-scale inhomogeneity and unsteadiness of the flow. In the following, it is shown that this limitation can be alleviated by considering an adaptive exponential smoothing, in which the smoothing factor $\alpha(\mathbf{x}, t)$ adjusts itself automatically according to the local turbulent rate of the velocity field. This procedure is made possible by means of an adaptive Kalman filter. Integrating Kalman filtering in the SISM therefore allows us to extend the scope of this sudgrid-scale model to the LES of inhomogeneous and unsteady turbulent flows.

\subsection{Adaptive exponential smoothing based on Kalman filtering}

A Kalman filter estimates the state of a dynamical system, here the low-frequency component of the velocity field, from a series of observations. Kalman filtering is a major topic in control theory in engineering science and is known to be rather efficient $[23,24]$. As for the exponential smoothing, an important feature of a Kalman filter is its formulation as a recursive estimator. The updated state is computed from the previous state and the current observation only. In our case, the update is made according to Eq. (2) but with a smoothing factor (noted $K$ ) that is now inferred dynamically from the local fluctuation of the signal. This inference is performed on the basis 
Table 1

The algorithm of our adaptive Kalman filter for turbulent flows. In the jargon of signal processing, $P$ is the error covariance and $K$ is the optimal Kalman gain. Notice that $K$ is formally equivalent to an adaptive smoothing factor according to Eq. (2): $K(\mathbf{x}, t) \simeq$ $\alpha(\mathbf{x}, t)$. InIT is the initialization step. The algorithm is recursive and organizes in three steps: PREDICT, UPDATE and ADAPT. In the ADAPT step, $\epsilon=0.1$ (recommended value) is an arbitrary small parameter.

\begin{tabular}{ll}
\hline INIT: & $\widetilde{\overline{\mathbf{u}}}^{(0)}=\overline{\mathbf{u}}^{(0)}$ \\
& $\sigma_{\delta \bar{u}}^{(0)}=\sigma_{\delta \tilde{\bar{u}}}$ with $\sigma_{\delta \tilde{\bar{u}}}$ given by Eq. (5) \\
& $P^{(0)}=\sigma_{\delta \bar{u}}^{(0)}$ \\
& $\widetilde{\overline{\mathbf{u}}}^{(n+1)}=\widetilde{\overline{\mathbf{u}}}^{(n)}$ \\
& $P^{(n+1)}=P^{(n)}+\sigma_{\delta \overline{\bar{u}}}^{2}$ with $\sigma_{\delta \tilde{\bar{u}}}$ given by Eq. (5) \\
PREDICT: & $K^{(n+1)}=P^{(n+1)} /\left(P^{(n+1)}+\sigma_{\delta \bar{u}}^{2(n)}\right)$ \\
& $\widetilde{\widetilde{\mathbf{u}}}^{(n+1)}=\left(1-K^{(n+1)}\right) \cdot \widetilde{\overline{\mathbf{u}}}^{(n+1)}+K^{(n+1)} \cdot \overline{\mathbf{u}}^{(n+1)}$ \\
UPDATE: & $P^{(n+1)}=P^{(n+1)} \cdot\left(1-K^{(n+1)}\right)$ \\
& $\sigma_{\delta \bar{u}}^{(n+1)}=\max \left(u^{*} \cdot\left\|\widetilde{\overline{\mathbf{u}}}^{(n+1)}-\overline{\mathbf{u}}^{(n+1)}\right\|, \epsilon \cdot u^{* 2}\right)$ \\
&
\end{tabular}

of a simple state-space representation of the velocity signal, as now explained.

The problem may be formulated quite simply as

$\left\{\begin{array}{l}\widetilde{\overline{\mathbf{u}}}^{(n+1)}=\widetilde{\overline{\mathbf{u}}}^{(n)}+\delta \widetilde{\overline{\mathbf{u}}}^{(n+1)} \\ \overline{\mathbf{u}}^{(n+1)}=\widetilde{\overline{\mathbf{u}}}^{(n+1)}+\delta \overline{\mathbf{u}}^{(n+1)}\end{array}\right.$

In this form, the smoothed velocity $\widetilde{\overline{\mathbf{u}}}$ evolves with some control increment $\delta \widetilde{\overline{\mathbf{u}}}$, and the second measurement equation expresses the deviation of the instantaneous velocity from the estimated lowfrequency component: $\delta \overline{\mathbf{u}}=\overline{\mathbf{u}}-\widetilde{\overline{\mathbf{u}}}$. As a first approximation, these two increments are considered as random Gaussian processes with zero mean. Furthermore, one may fairly assume that the evolution of the smoothed velocity is very slow compared to the evolution of the instantaneous velocity, so that no deterministic evolution is prescribed at first for $\widetilde{\mathbf{u}}$, i.e. $\widetilde{\overline{\mathbf{u}}}^{(n+1)}=\widetilde{\overline{\mathbf{u}}}^{(n)}$ a priori. However, $\widetilde{\overline{\mathbf{u}}}^{(n)}$ is allowed to evolve (slowly) if one observes that the instantaneous value departs significantly from the smoothed signal. The Kalman filter enters a correction stage to determine $\delta \widetilde{\mathbf{u}}^{(n+1)}$. To do so, the variance of $\delta \widetilde{\mathbf{u}}^{(n+1)}$ is considered as fixed for the whole flow, whereas the variance of $\delta \overline{\mathbf{u}}^{(n+1)}$ is re-evaluated at each grid point as the simulation progresses. This assumption is specific to our modeling since both variances should be considered as constant in the standard theory of Kalman filter. Here, the physical idea is to assume that the instantaneous velocity is allowed to depart more or less from its low-frequency component depending on the local turbulence rate, whereas the range of variation of the low-frequency component is prescribed as a characteristic of the flow (see below). This feature is essential to track possible instabilities in the flow.

More specifically, the standard deviation of $\delta \widetilde{\overline{\mathbf{u}}}$ is kept constant throughout the iterations with

$\sigma_{\delta \tilde{\mathbf{u}}}=\frac{2 \pi f^{*} \Delta t}{\sqrt{3}} u^{*}$,

where the frequency $f^{*}$ and velocity $u^{*}$ represent characteristic values of the flow. This specific choice for $\sigma_{\delta \widetilde{\overline{\mathbf{u}}}}$ stems from the property that, in a steady regime, our Kalman filter should behave as an exponential smoothing with $\alpha=\sigma_{\delta \widetilde{\overline{\mathbf{u}}}} / \sigma_{\delta \overline{\mathbf{u}}}$ [8]. By assuming that $\sigma_{\delta \overline{\mathbf{u}}} \simeq u^{*}$, this leads to $\alpha \simeq 2 \pi f^{*} \Delta t / \sqrt{3}$ which is fully consistent with Eq. (3) by identifying $f^{*}$ with the cut-off frequency $f_{c}$.

\subsection{Implementation and setting of the Kalman filter}

The theory of Kalman filter applied to our modeling leads to the algorithm sketched in Table 1. In practice, this algorithm is applied to the velocity vector at each grid point. The low-frequency part of the rate of strain is obtained by deriving spatially the smoothed velocity, since derivation and smoothing are linear operations that commute.
The ADAPT step is a key point of our algorithm. In order to make the Kalman filter adaptive, an estimate of the variance of $\delta \bar{u}$ is required at each time step. For this purpose, we introduce the estimator

$\sigma_{\delta \bar{u}}^{2(n+1)}=\max \left(u^{*} \cdot\left\|\widetilde{\overline{\mathbf{u}}}^{(n+1)}-\overline{\mathbf{u}}^{(n+1)}\right\|, \epsilon \cdot u^{* 2}\right)$

in which $\epsilon$ is an arbitrary small parameter

The first term within the maximum is ad-hoc and is devised to yield a physically-sound estimation of the velocity variance: $u^{*}$ is fixed while $\left\|\widetilde{\mathbf{u}}^{(n+1)}-\overline{\mathbf{u}}^{(n+1)}\right\|$ takes into account the observed (instantaneous) fluctuation. The second term is a lower bound that prevents $\sigma_{\delta \bar{u}}^{2}$ from decreasing to zero. Otherwise, if $\sigma_{\delta \bar{u}}^{2(n)} \approx 0$ then $K^{(n+1)} \approx 1$ and $\widetilde{\overline{\mathbf{u}}}^{(n+1)} \approx \overline{\mathbf{u}}^{(n+1)}$. At the next time step, $\sigma_{\delta \bar{u}}^{2(n+1)} \approx 0$ and so on. The filtered signal then sticks to the instantaneous signal for all the subsequent time steps. In order to avoid this pitfall, the value $\epsilon \cdot u^{* 2}$ acts as a small but non-zero lower bound for $\sigma_{\delta \bar{u}}^{2}$. A recommended value for $\epsilon$ is 0.1 according to [8]. This bound is reached in regions where the flow is laminar, and permits to efficiently track the onset of turbulence. When fluctuations grow, the estimated observation variance $\sigma_{\delta \bar{u}}^{2(n)}$ increases and $K^{(n+1)}$ decreases, etc. The sensitivity to the value of the parameter $\epsilon$ will be examined in the test case.

The memory usage of our Kalman algorithm remains very low since it only requires the storage of the error covariance, $P^{(n)}$, the variance, $\sigma_{\delta \bar{u}}^{2(n)}$, and the value of the filtered signal, $\widetilde{\overline{\mathbf{u}}}^{(n)}$, at each grid point. The number of operations remains relatively small as well. Importantly, the calibration of the Kalman filter requires to specify the values of $u^{*}$ and $f^{*}$ in Eqs. (5) and (6). In practice, a fine tuning of $u^{*}$ and $f^{*}$ should not be necessary because the Kalman filter is expected to self-adjust, and thus, characteristic values are sufficient. Nevertheless, the sensitivity to the value of $f^{*}$ will be addressed in the test case.

The values $u^{*}$ and $f^{*}$ are typically obtained from the characteristic values entering in the Reynolds number or other flow numbers, e.g. the Strouhal number. For instance, for a turbulent plane-channel flow: $\operatorname{Re}_{\tau}=u_{\tau} \cdot h / v$, where $u_{\tau}$ is the wall-friction velocity, $h$ is the half-width of the channel and $v$ is the kinematic viscosity of the fluid. A relevant choice is therefore $u^{*}=u_{\tau}$ and $f^{*}=u_{\tau} / h$. Likewise, relevant values for $u^{*}$ and $f^{*}$ will be proposed for the flow past a cylinder in the following section.

\section{Flow past a circular cylinder in the subcritical turbulent regime}

\subsection{Numerical solver}

Our computations have been carried out with the TuRB'Flow solver [25], which is primarily devoted to compressible aerodynamics in the domain of turbomachinery flows. Spatial discretization uses finite volumes on multiblock structured grids. The estimation of the inviscid fluxes relies on a four-point centered interpolation with fourthorder artificial viscosity (coefficient is equal to 0.01 [26]), whereas diffusive fluxes are computed with a two-point centered scheme. The time marching is carried out by a five-step Runge-Kutta scheme. Details about the flow equations and the solver are reported in [25]. In the present study, the subgrid-scale viscosity follows the prescription of the shear-improved Smagorinsky model (Eq. (1)) with the lowfrequency component of the rate of strain being estimated by means of the adaptive Kalman filter described in Section 2.

\subsection{Flow configuration}

The flow past a cylinder is considered in the subcritical regime where shear-layer transition occurs just downstream of the separation points [27]. The complexity of the flow arises from the oscillation of the separation points along the cylinder sides, related to 

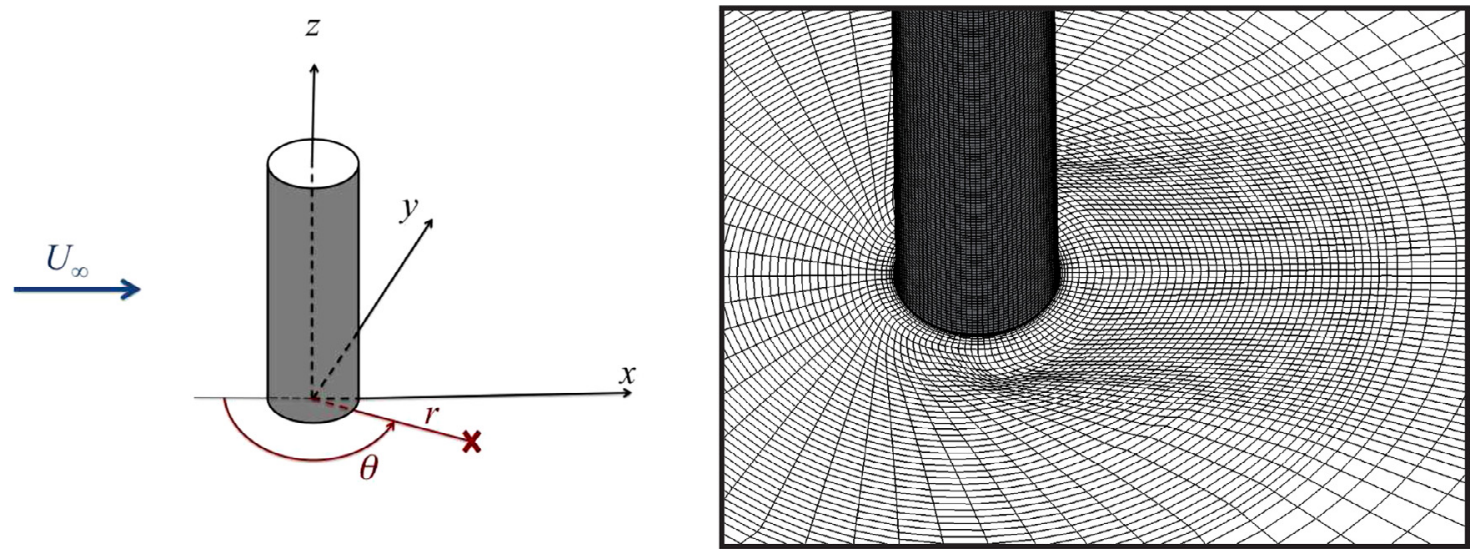

Fig. 1. Left: sketch of the Cartesian coordinate system $(x, y, z)$ and the cylindrical coordinate system $(r, \theta, z)$. Right: close-up view of the mesh around the cylinder. For clarity, every other point is displayed in each direction.

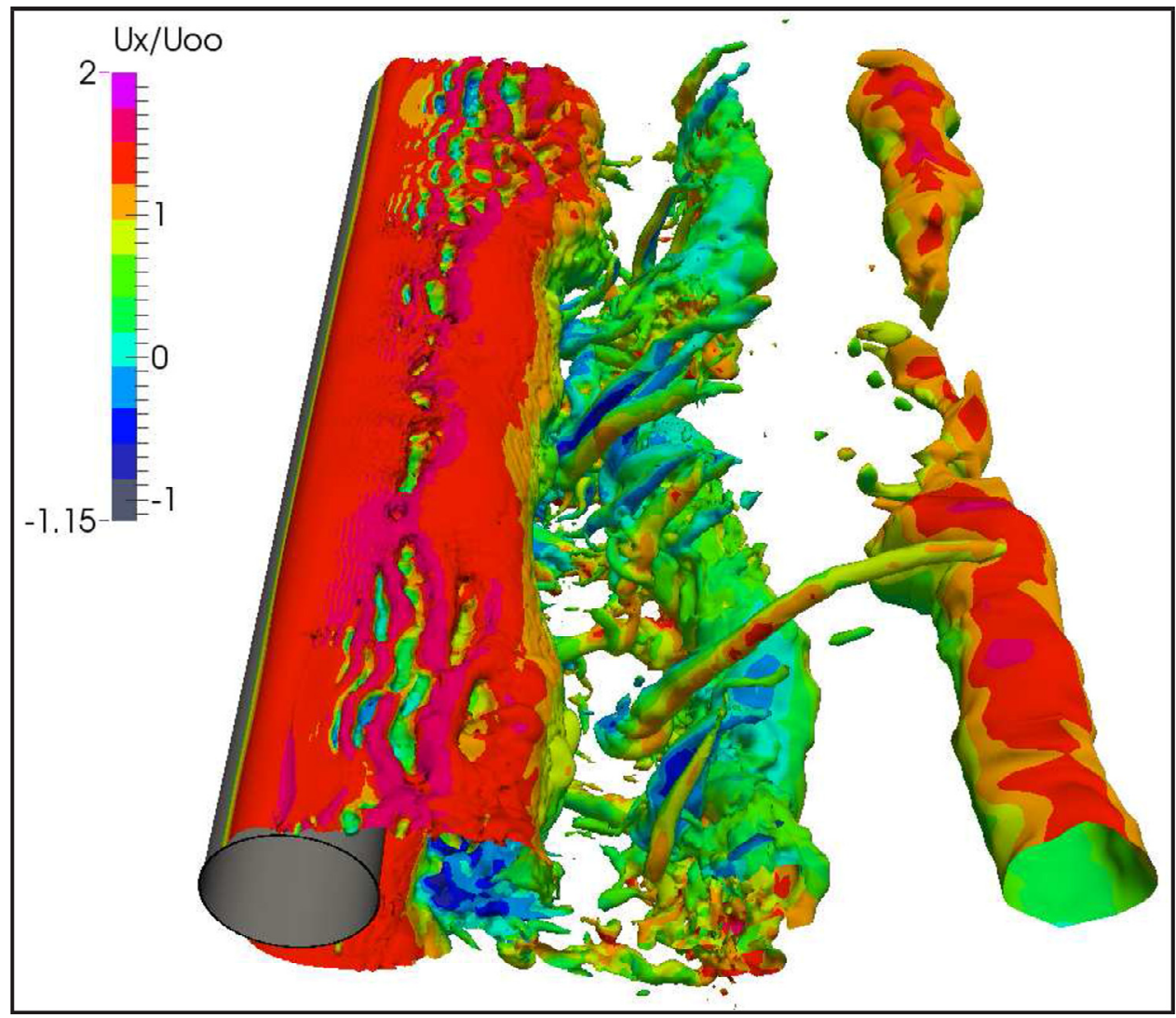

Fig. 2. Instantaneous iso-surface of density, colored by non-dimensional axial velocity $u_{x} / U_{\infty}$

the vortex shedding in interaction with background wake turbulence. From the viewpoint of LES, this flow is recognized as a challenging test case $[28,29]$. It is here used to test the efficiency of our smoothing algorithm and the capability of the shear-improved Smagorinsky model to handle complex unsteady flows. Another advantage is the large body of available experimental results at comparable Reynolds numbers that allow us to test in detail the accuracy of our numerical simulations. The robustness of the method will also be examined by varying the parameters entering in the calibration of the Kalman procedure.

The numerical configuration is a circular cylinder (of axis $z$ ) with diameter $D=0.01 \mathrm{~m}$ and span length $9 D$, which is placed in an undisturbed air-flow at velocity $U_{\infty}=70 \mathrm{~m} . \mathrm{s}^{-1}$ along the $x$-direction (under standard conditions of temperature and pressure). In this configuration, the Reynolds number is $\operatorname{Re}=4.7 \times 10^{4}$ based on the diameter. Vortices are shed from each side of the cylinder at a Strouhal number $\mathrm{St}=f_{S} \cdot D / U_{\infty} \approx 0.2$ corresponding to the vortexshedding frequency $f_{s} \approx 1400 \mathrm{~Hz}$. The calibration of the Kalman filter requires characteristic values associated with the largest scales of the flow: $u^{*}$ and $f^{*}$. In our case, the largest scales are associated with the vortex shedding and a natural choice is therefore $u^{*}=U_{\infty}$ and $f^{*}=f_{s}$ (Run-A). In order to estimate the sensitivity to the value of $f^{*}$, a second simulation will be performed with the overestimated value $f^{*}=2 f_{s}$ (Run-B). Finally, a third simulation (Run-C) will be considered with $\epsilon=0.2$ in Eq. (6) instead of the recommended value $\epsilon=0.1$ [8]. RunA should be considered as our reference simulation, whereas Run$\mathrm{B}$ and Run-C are expected to be less favourable configurations. The sensitivity to the grid resolution (which fulfills usual standards for 
Table 2

Characteristic numbers of the flow for Run-A, Run-B and Run-C in comparison with various experimental data at similar Reynolds numbers. Run-A is the reference simulation with the calibration parameters $u^{*}=U_{\infty}, f^{*}=f_{s}$ and $\epsilon=0.1$, whereas $f^{*}=2 f_{s}$ in Run-B and $\epsilon=0.2$ in Run-C (other parameters are unchanged).

\begin{tabular}{|c|c|c|}
\hline & LES at $\operatorname{Re}_{D}=4.7 \times 10^{4}$ & experimental data found in the literature \\
\hline$C_{D}$ : mean drag coefficient & $\begin{array}{l}1.196 \pm 0.002 \text { (Run-A) } \\
1.216 \pm 0.006 \text { (Run-B) } \\
1.191 \pm 0.010 \text { (Run-C) } \\
0.036 \pm 0.001 \text { (Run-A) }\end{array}$ & $\begin{array}{l}1.35 \text { at } \operatorname{Re}=4.3 \cdot 10^{4}[30] \\
{[1.0,1.35] \text { at } \operatorname{Re}=4.8 \cdot 10^{4}[32]} \\
{[1.0,1.3] \text { at } \operatorname{Re}=4.8 \cdot 10^{4}[33]} \\
{[1.1,1.3] \text { at } 10^{4}<\operatorname{Re}<10^{5}[34]} \\
0.16 \text { at } \operatorname{Re}=4.3 \cdot 10^{4}[30]\end{array}$ \\
\hline $\mathrm{C}_{\mathrm{D}}^{\prime}$ : rms drag coefficient & $\begin{array}{l}0.048 \pm 0.003 \text { (Run-B) } \\
0.057 \pm 0.003 \text { (Run-C) } \\
0.48 \pm 0.01 \text { (Run-A) }\end{array}$ & $\begin{array}{l}{[0.08,0.1] \text { at } \operatorname{Re}=4.8 \cdot 10^{4}[35]} \\
{[0.05,0.1] \text { at } 10^{4}<\operatorname{Re}<10^{5}[34]} \\
{[0.45,0.55] \text { at } \operatorname{Re}=4.3 \cdot 10^{4}[30]}\end{array}$ \\
\hline $\mathrm{C}_{\mathrm{L}}^{\prime}:$ rms lift coefficient & $\begin{array}{l}0.52 \pm 0.02 \text { (Run-B) } \\
0.45 \pm 0.03 \text { (Run-C) } \\
0.20 \text { (Run-A) }\end{array}$ & $\begin{array}{l}{[0.4,0.8] \text { at } \operatorname{Re}=4.8 \cdot 10^{4}[35]} \\
{[0.6,0.82] \text { at } 10^{4}<\operatorname{Re}<10^{5}[34]} \\
{[0.18,0.2] \text { at } \operatorname{Re}=4.8 \cdot 10^{4}[32]}\end{array}$ \\
\hline St: Strouhal number & $\begin{array}{l}0.20 \text { (Run-B) } \\
0.20 \text { (Run-C) } \\
86.8^{\circ} \text { (Run-A) }\end{array}$ & {$[0.185,0.195]$ at $\operatorname{Re}=6.1 \cdot 10^{4}[36]$} \\
\hline$\theta_{s}:$ mean separation angle & $\begin{array}{l}86.7^{\circ}(\text { Run-B) } \\
85.4^{\circ}(\text { Run }-\mathrm{C}) \\
-1.27(\text { Run-A) }\end{array}$ & $\begin{array}{l}83^{\circ} \text { at } 4.010^{4}<\operatorname{Re}<4.510^{4}[34] \\
{[-1.38,-1.22] \text { at } \operatorname{Re}=4.6 \cdot 10^{4}[38]}\end{array}$ \\
\hline $\begin{array}{l}C_{p \text { base }}: \text { mean base pressure coefficient } \\
C_{p \text { base }} \equiv C_{p}\left(\theta=180^{\circ}\right)\end{array}$ & $\begin{array}{l}-1.30 \text { (Run-B) } \\
-1.25 \text { (Run-C) } \\
1.24 \text { (Run-A) }\end{array}$ & 1.25 at $\operatorname{Re}=4.7 \cdot 10^{4}[40]$ \\
\hline $\begin{array}{l}\ell_{c} / D \text { : mean recirculation-bubble length } \\
\text { normalized by the diameter of the cylinder }\end{array}$ & $\begin{array}{l}1.19 \text { (Run-B) } \\
1.26 \text { (Run-C) }\end{array}$ & \\
\hline $\begin{array}{l}u_{\operatorname{rev}_{\max }} / U_{\infty}: \text { maximum of mean reverse velocity } \\
\text { normalized by the inlet velocity }\end{array}$ & $\begin{array}{l}-0.28 \text { (Run-A) } \\
-0.26 \text { (Run-B) } \\
-0.28 \text { (Run-C) }\end{array}$ & \\
\hline
\end{tabular}

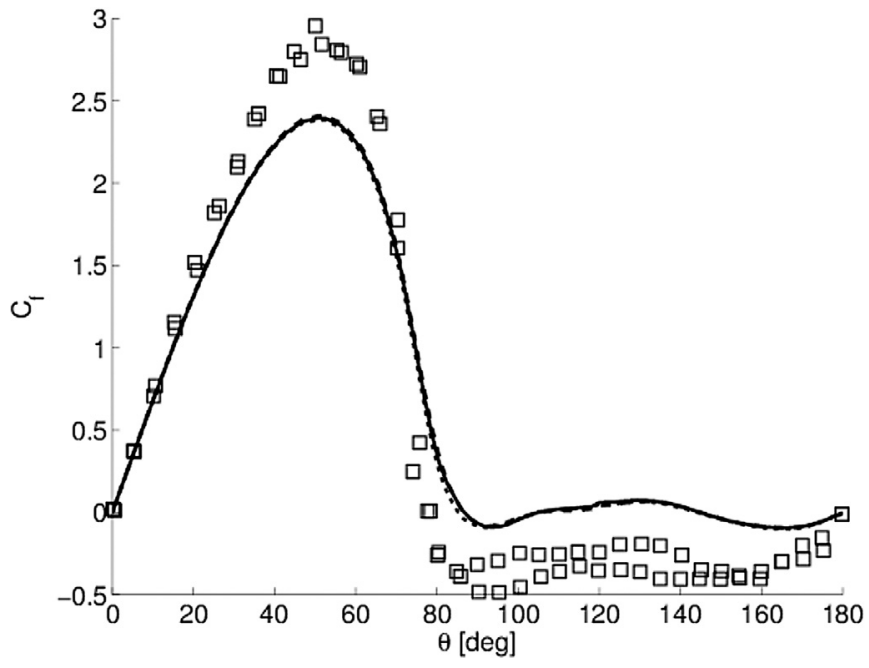

Fig. 3. Mean friction coefficient around the cylinder. LES at $\operatorname{Re}=4.7 \cdot 10^{4}$ (Run-A: solid line, Run-B: dashed line, Run-C: dotted line) are compared with experimental data ( $\square$ : $\left.\operatorname{Re}=10^{5}[33]\right)$.

wall-resolved LES) is not examined here since the filtering procedure operates in the time domain only, and is thus not directly affected by the spatial resolution.

The aspect ratio $L / D$ has a strong impact on the physics of this flow, as reported in [30]. In particular, $L / D>2.5$ is required to observe a regular vortex-shedding, however, for $L / D<4$ the mean drag and fluctuating lift are anomalously increased. Therefore, in the present simulations, $L / D=9$ and periodicity is imposed in the spanwise direction. The computational domain extends over $20 \mathrm{D}$ in the radial direction. The coordinate system and a view of the grid are presented in Fig. 1. The whole grid is composed of about $9 \cdot 10^{6}$ points. The resolution is consistent with standard practices for (wall-resolved) LES of engineering flows [3]. Behind the separation line (in the

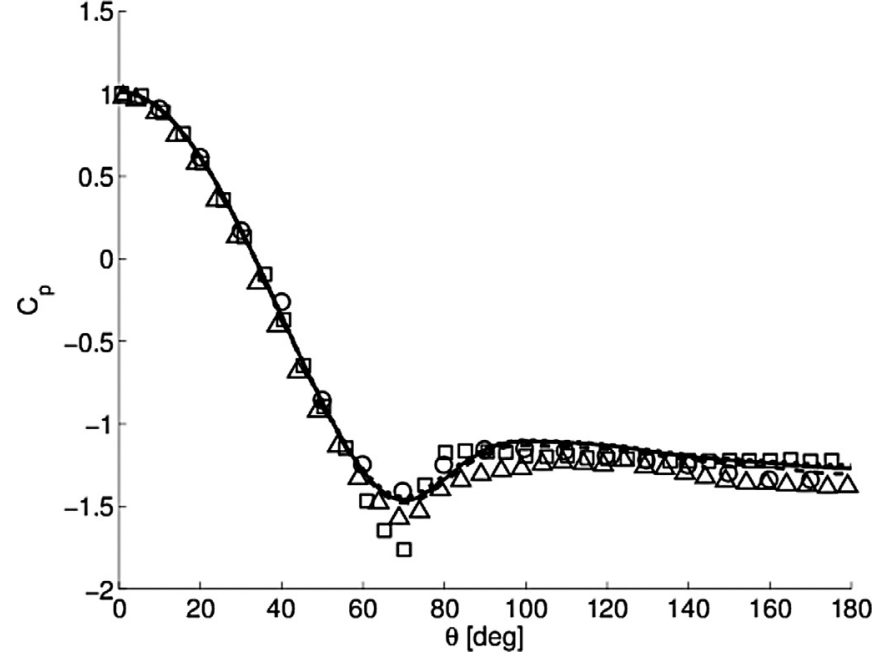

Fig. 4. Mean pressure coefficient around the cylinder. LES at $\mathrm{Re}=4.7 \cdot 10^{4}$ (Run-A: solid line, Run-B: dashed line, Run-C: dotted line) are compared with experimental data $\left(\bigcirc: \operatorname{Re}=4.6 \cdot 10^{4}[38], \square: \operatorname{Re}=10^{5}[33], \triangle: \operatorname{Re}=1.3 \cdot 10^{5}[30]\right)$.

turbulent region) the wall grid density in wall units is $\Delta r_{\max }^{+} \simeq 1$ (with a radial growth ratio of 1.2), $r^{+} \Delta \theta_{\max }^{+} \simeq 15$ and $\Delta z_{\max }^{+} \simeq 22$. The non-dimensionnal time step is $\Delta t^{+}=\Delta t \cdot U_{\infty} / D=4.2 \times 10^{-4}$, adapted to explicit discretization. For each run, about 1340 flow instants have been recorded in the established turbulent regime to ensure a good convergence of the statistics; the whole trace corresponds to 67 vortex-shedding periods. In the following text $\langle$.$\rangle denotes the$ post-processed time average.

\section{Results and analysis}

In order to give an overview of the flow structure, an instantaneous iso-surface of density, colored by the non-dimensional axial 


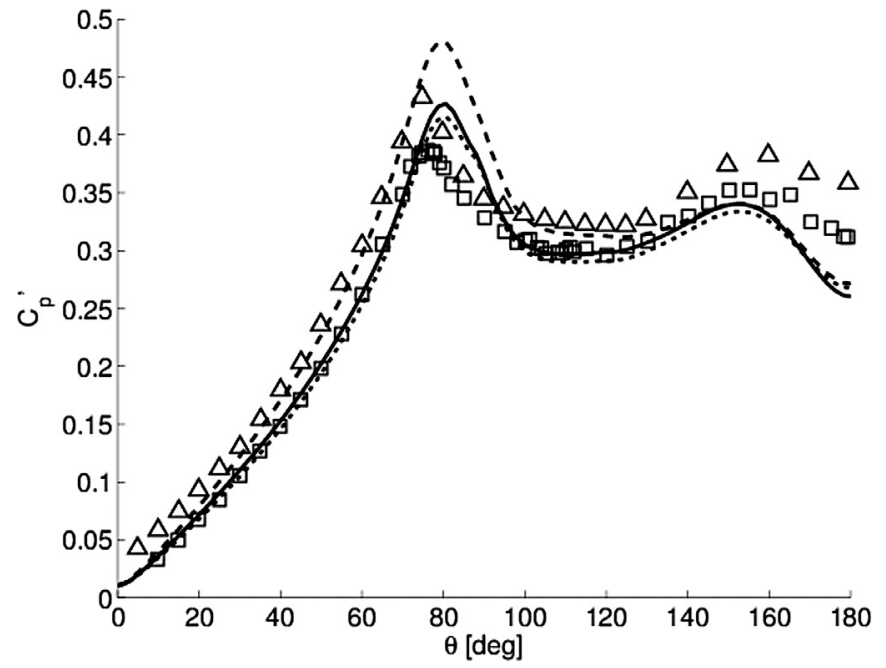

Fig. 5. Root-mean-squared pressure coefficient around the cylinder. LES at $\operatorname{Re}=4.7$. $10^{4}$ (Run-A: solid line, Run-B: dashed line, Run-C: dotted line) are compared with experimental data $\left(\square: \operatorname{Re}=6.1 \cdot 10^{4}[36] ; \triangle: \operatorname{Re}=6.1 \cdot 10^{4}[39]\right)$.

velocity $u_{x} / U_{\infty}$, is displayed in Fig. 2 . Three large vortices are observed in the wake, shed from the cylinder during the previous iterations, with alternate senses of rotation. These vortices are affected by turbulence and distorted in the spanwise direction. Axial vortices are also observed between successive shed vortices. Upstream, in the shear layers separating from the sides of the cylinder, smaller spanwise vortices are observed. According to [31], they may be generated by the nascent shear-layer instability in the early transition process.

\subsection{Aerodynamical characteristics}

The different characteristic numbers of the flow obtained from our simulations are gathered together in Table 2 and compared with various experimental data at comparable Reynolds numbers. For the force coefficients: $C_{D}, C_{D}{ }^{\prime}$ and $C_{L}{ }^{\prime}$, an estimate of the uncertainty of the statistical convergence is provided. It is evaluated as the standard deviation of the coefficient when calculated on subsets of the whole sample. These subsets are obtained by masking 17 vortex-shedding periods from the total sample (67 periods) and by sliding the mask.

The overall comparison is very satisfactory and all estimated values lie within, or are close to, the ranges of experimental results. Note that the different Reynolds numbers and aspect ratios contribute to the dispersion of the reported experimental data. Except for the fluctuating drag coefficient, $C_{D}{ }^{\prime}$, one gets that the results obtained with a different reference frequency $f^{*}$ (Run-B) or a different parameter $\epsilon$ (Run-C) remain very close to the results obtained in the reference simulation (Run-A). The differences between the simulations are of the same order as the uncertainties on the statistical convergence. This underlines a good robustness of our numerical method. However, large discrepancies are reported on $C_{D}{ }^{\prime}$ between the three simulations, in terms of percentage or by comparison with the uncertainties on the convergence. But, one can mention that this coefficient is very small (a few percents of the mean drag) and is probably also more sensitive to computational errors.

The pressure distribution around the cylinder is strongly influenced by the vortex shedding so that wall-pressure spectra are dominated by a peak at the vortex-shedding frequency (except near $\theta=0^{\circ}$ and $\theta=180^{\circ}$ ). The Strouhal number is calculated from this peak frequency measured on one side of the cylinder $\left(\theta=90^{\circ}\right)$. It is found in very good agreement with the experimental data (see Table 2 ). In Fig. 3, the angular distribution of the mean friction coefficient is given

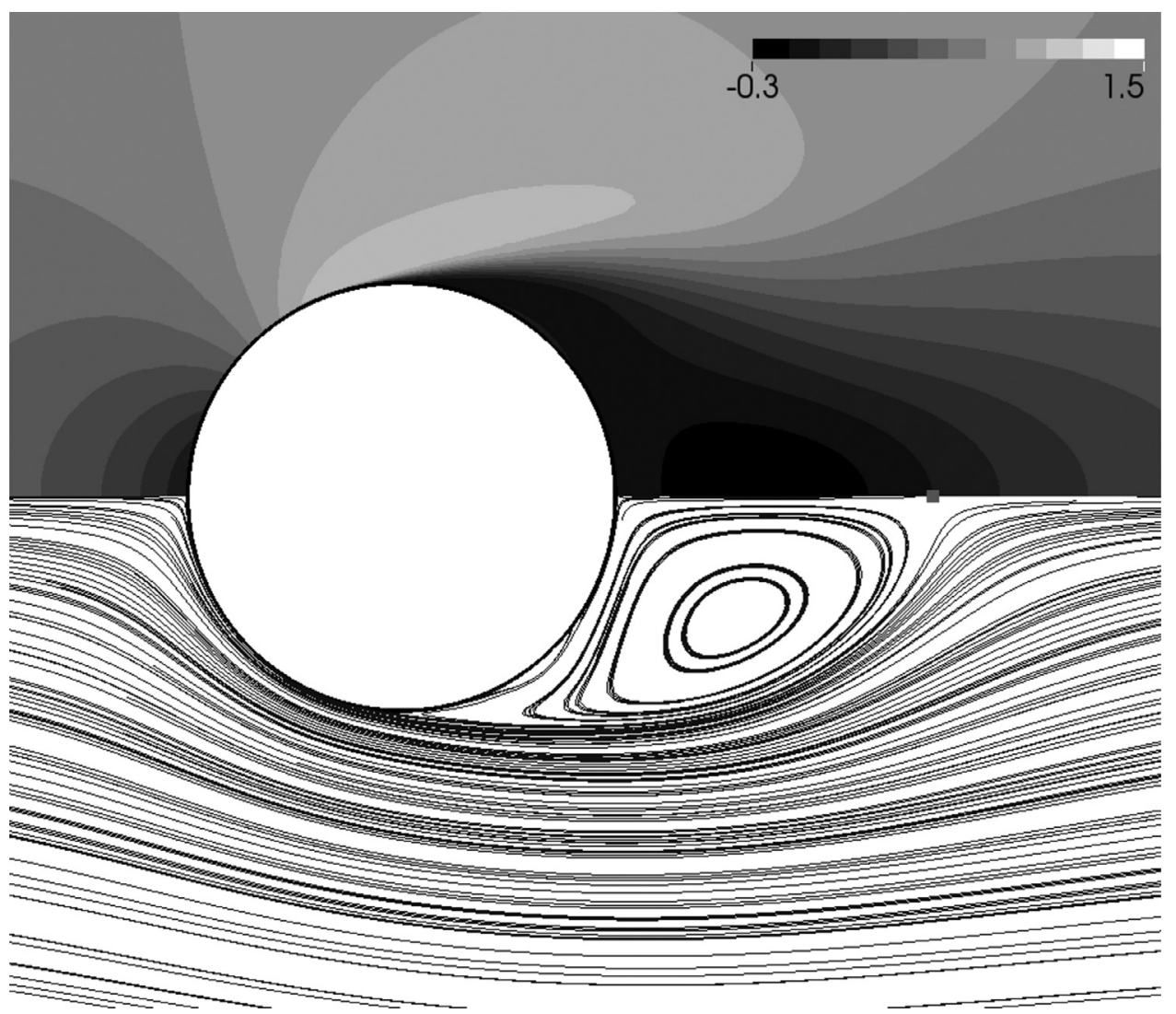

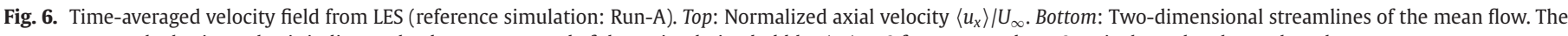
grey square on the horizontal axis indicates the downstream end of the recirculation bubble: $\left\langle u_{x}\right\rangle=0$ for $x=\ell_{c}$ and $y=0$; $\ell_{c}$ is the wake-closure length. 

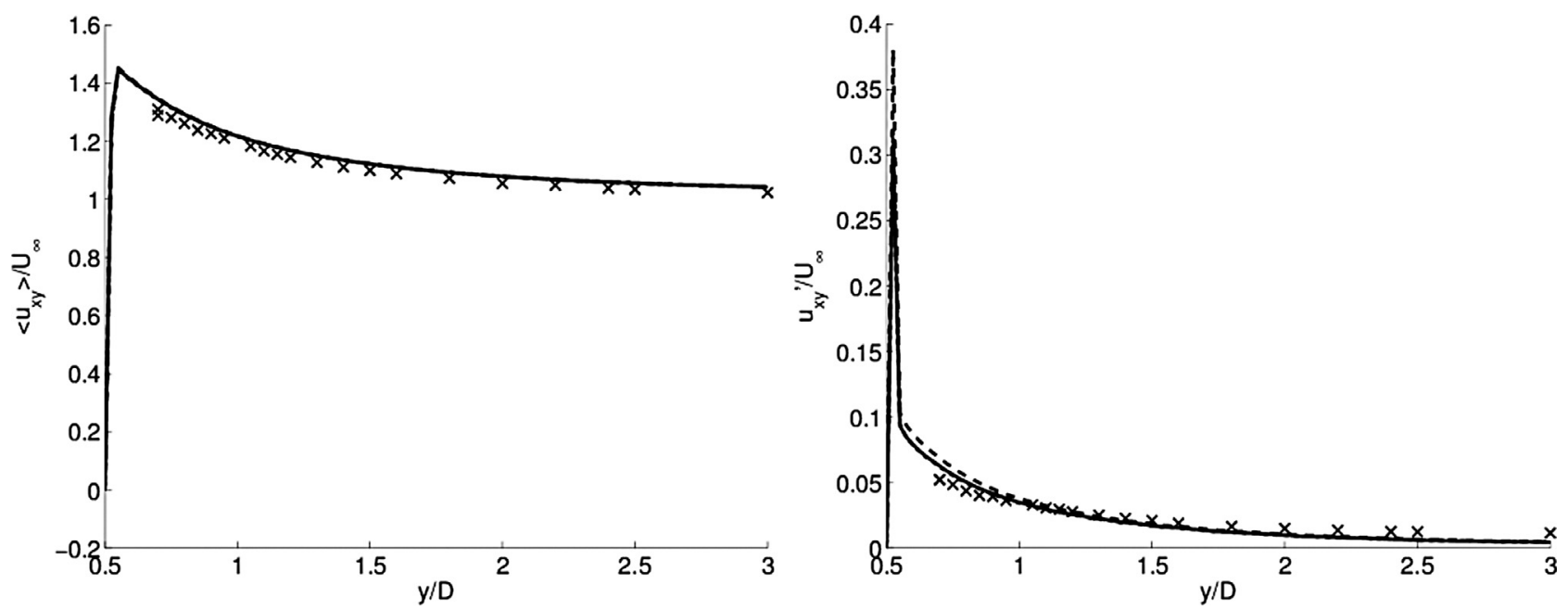

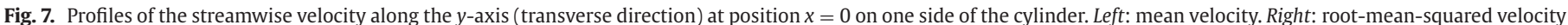
fluctuations. - : LES at Re $=4.7 \times 10^{4}$ (Run-A: solid line, Run-B: dashed line, Run-C: dotted line); $\times$ : experimental data at Re $=4.7 \times 10^{4}[41]$.
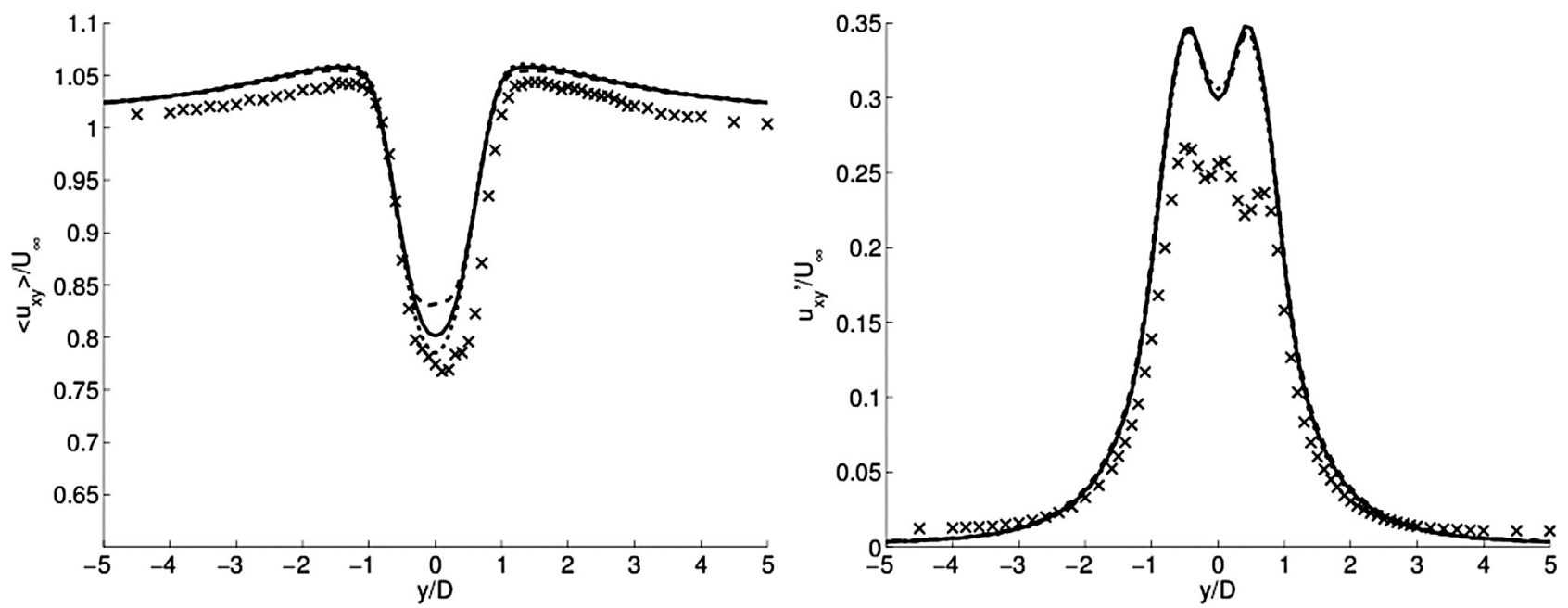

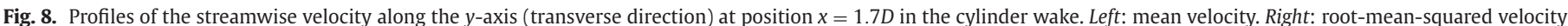
fluctuations. - : LES at Re $=4.7 \times 10^{4}$ (Run-A: solid line, Run-B: dashed line, Run-C: dotted line); $\times:$ experimental data at Re $=4.7 \times 10^{4}[41]$.
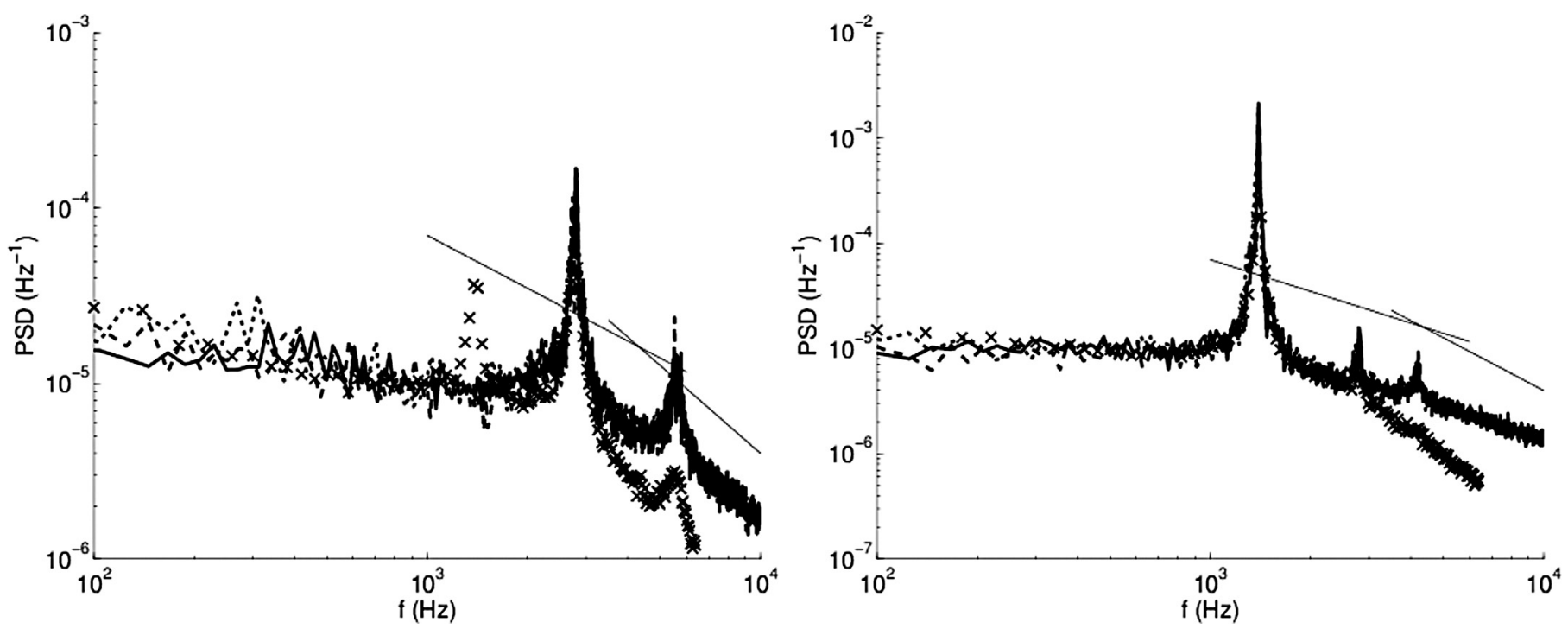

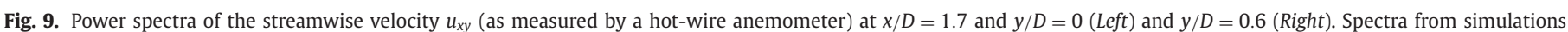

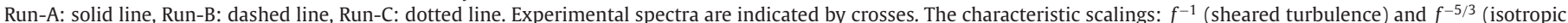
turbulence) are shown for indication. 


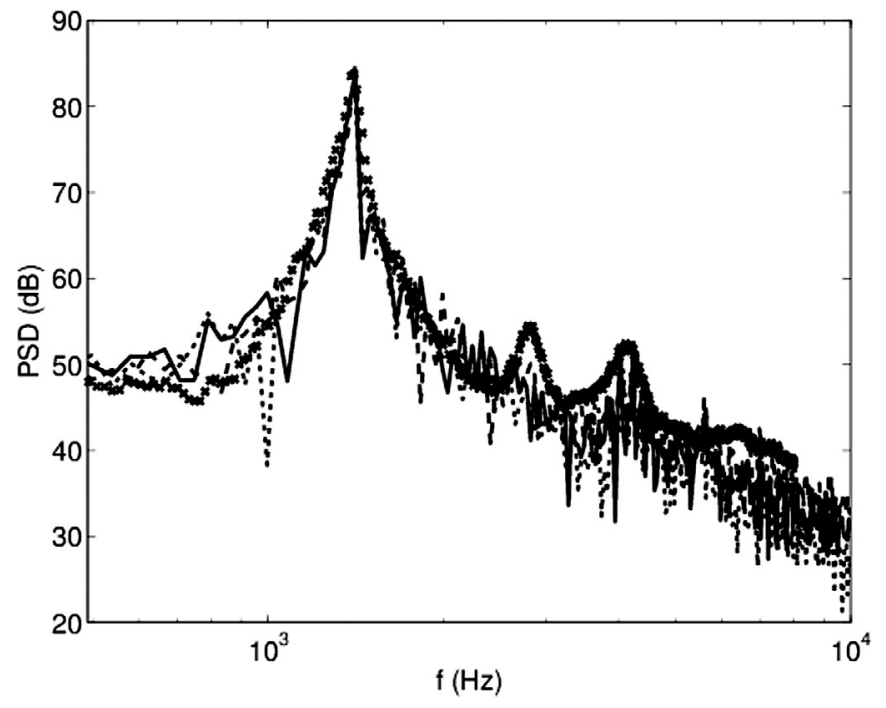

Fig. 10. Far-field power spectrum of the pressure (in $\mathrm{Pa}^{2} . \mathrm{Hz}^{-1}$ ) represented in decibels with reference pressure $2 \cdot 10^{-5} \mathrm{~Pa}$, at position $x / D=15.5$ and $y / D=185$. Spectra from simulations Run-A: solid line, Run-B: dashed line, Run-C: dotted line. Experimental spectrum is indicated by crosses.

by $C_{f} \equiv\left(\left\langle\tau_{w}\right\rangle / \rho U_{\infty}^{2}\right) \sqrt{\operatorname{Re}}$, where $\tau_{w}$ is the shear stress around the cylinder. It agrees well with the experimental data for the three simulations. In Table 2 , the mean separation angle is given by $C_{f}=0$. For the reference simulation (Run-A), the computed value is $\theta_{s}=86.8^{\circ}$ close to the experimental value $\theta_{s} \approx 83^{\circ}$ reported in [34] within the range $4.0 \cdot 10^{4} \leq \operatorname{Re} \leq 4.5 \cdot 10^{4}$. The over-estimate of $\theta_{s}$ is typically of the order of the angular resolution $\Delta \theta \approx 2^{\circ}$ in the separation zone. Interestingly, no notable discrepancy is observed for $\theta_{s}$ between the three simulations, although the parameter $\epsilon$ is expected to play an important role in capturing the onset of turbulence (near the separation point). This indicates that $\epsilon=0.1$ is certainly the good order of magnitude [8].

In Fig. 4 , the angular distribution of the mean pressure coefficient is given by $C_{p} \equiv\left(\langle p\rangle-p_{\infty}\right) / \frac{1}{2} \rho U_{\infty}^{2}$ where $p_{\infty}$ is the ambient pressure imposed at the outflow. The prediction is again satisfactory without clear discrepancy between the three runs. The distribution of the fluctuating pressure coefficient $C_{p}^{\prime} \equiv p_{\text {rms }} / \frac{1}{2} \rho U_{\infty}^{2}$ is plotted in Fig. 5. This quantity is particularly sensitive to the turbulent wake dynamics. In the reference simulation (Run-A), it is remarkably well captured, with a maximum located near the separation angle [37]. The results of Run-A and Run-C are almost indistinguishable, which means that varying the parameter $\epsilon$ does not affect significantly the computation of wake dynamics. A slight discrepancy (of the order of $10 \%$ ) is observed around the maximum when over-estimating the characteristic frequency $f^{*}$ (Run-B). Indeed, over-estimating $f^{*}$ is expected to result in an under-estimate of the subgrid-scale viscosity, since this latter is typically proportional to the difference between the instantaneous and the filtered shear. Turbulent fluctuations, and consequently $C_{p}^{\prime}$, are therefore increased.

The flow downstream of the cylinder is examined herein. The mean velocity in the plane perpendicular to the cylinder is plotted in Fig. 6. The mean recirculation zone (or bubble) can be seen on the streamline plot and is characterized by the wake-closure length, $\ell_{c}$, where the centerline mean axial velocity vanishes: $\left\langle u_{x}\left(\ell_{c}, 0\right)\right\rangle=0$. The reference simulation (Run-A) yields $\ell_{c} \approx 1.24 D$, which is very close to the experimental value $1.25 D$ at the same Reynolds number [40]. The estimation obtained in Run-C, $\ell_{c} \approx 1.26 D$, is also very close to the experimental value, whereas the discrepancy obtained in Run-B is much larger: $\ell_{c} \approx 1.19 D$. This is consistent with our previous observations, namely that the sensitivity to the reference frequency $f^{*}$ appears to be higher than to the parameter $\epsilon$, however, the sensitivity remains relatively low for these two quantities.

\subsection{Flow statistics in the wake}

In Figs. 7 and 8, comparisons with hot-wire velocity profiles [41] are plotted at two positions: (i) aside the cylinder, shortly downstream of the separation point $(x=0)$ and (ii) in the near wake downstream of the recirculation bubble $(x / D=1.7)$. The mean and fluctuating velocity components are those directly measured by the hot-wire anemometer and evaluated accordingly from the LES data. Namely, the hot-wire yields an estimation of the norm of the velocity in the plane perpendicular to the cylinder axis: $u_{x y} \equiv \sqrt{u_{x}^{2}+u_{y}^{2}}$. The mean and root-mean-squared velocity profiles at $x=0$ match reasonably well the experimental results. In the near wake (at $x=1.7 D$ )
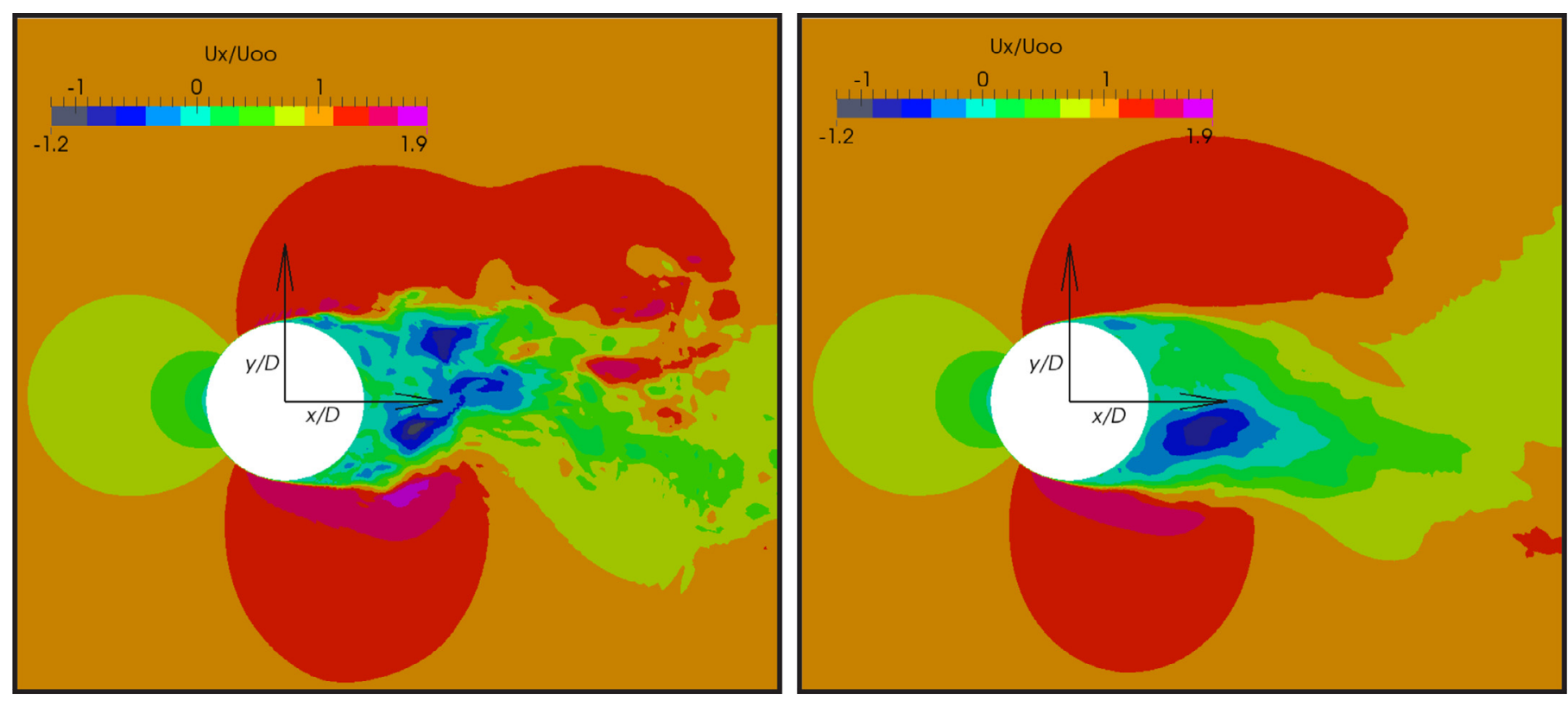

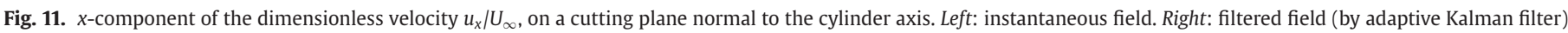
at the same instant. Results from Run-A. The unit vectors of the coordinate system $(x / D, y / D)$ are shown. 


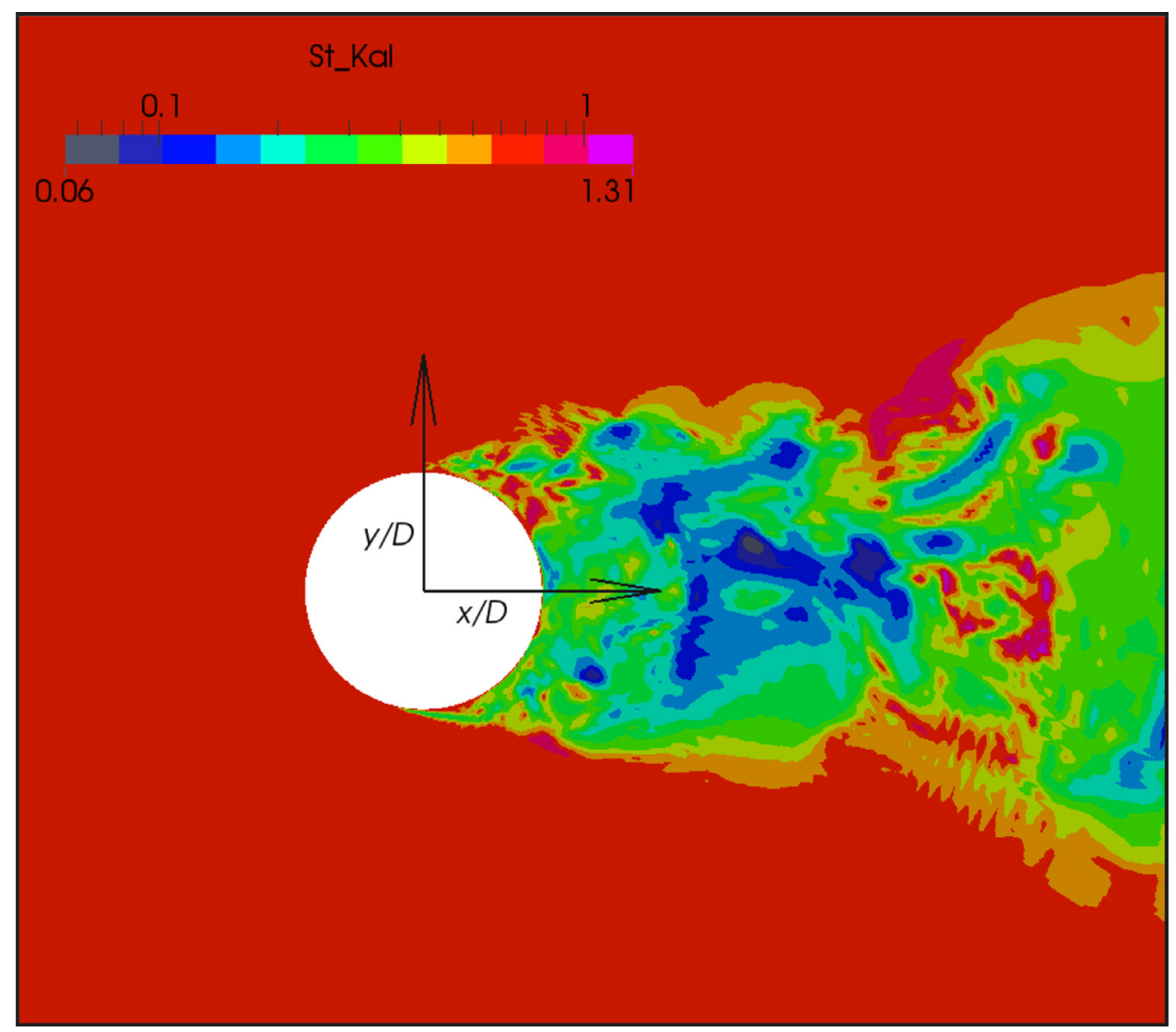

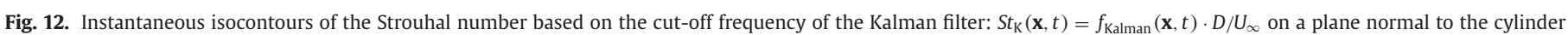
axis, at the same position and time as in Fig. 11. Results from Run-A. The unit vectors of the coordinate system $(x / D, y / D)$ are shown.

the overall agreement is quite good, although a discrepancy is found for both the mean velocity and velocity fluctuations. This region, in the near wake downstream of the recirculation bubble, is particularly sensitive. Indeed, it is affected by the wake closure length and undergoes violent turbulent events. In this regard, the prediction here achieved is encouraging. Moreover, it should be pointed out that hotwire measurement might also be biased (downstream of the mean recirculation bubble) by backflow events. Indeed, whenever $u_{x}<0$, the hot wire is in the own wake of the probe and underestimates $u_{x y}$. For this reason, standard single hot-wire is not expected to provide a precise measurement of the velocity in the near wake [30]. This issue has been tackled by Cantwell and Coles [32] by rotating X-array hot-wire probes through the measurement domain, however, such advanced measurement technique has not been considered here. Finally, let us mention that there is no notable discrepancy between the three runs.

\subsection{Spectral analysis}

In order to assess the quality of our LES, it is important to investigate the unsteady part of the fluctuating fields as well. Since the flow is subject to both a quasi-periodic vortex shedding and broadband turbulence, a deeper insight into statistics is provided by a spectral analysis. In this subsection various spectra are examined, namely, velocity spectra in the cylinder near wake and a far-field pressure spectrum.

Fig. 9 displays the power spectra of the streamwise velocity $u_{x y}$ obtained both experimentally and numerically at $x / D=1.7$ and two vertical positions $y=0$ and $y / D=0.6$, i.e. on the centerline and in the shear layer of the near wake, respectively. The general features of the spectra are in line with the standard aerodynamical description of subcritical von-Karman streets [34]. In the near wake, experimental velocity signals fluctuate almost sinusoidally at the vortex-shedding frequency $f_{s} \approx 1400 \mathrm{~Hz}$. Deviations from the purely periodic signal are evidenced by the broadband part of the spectrum, including the spectral broadening of the tonal components. The numerical results follow the same trends, although the signals are characterized by a richer harmonic content (second and third harmonics).

The peak at the fundamental frequency (at $f_{s}$ ) is absent in the simulated spectra on the centerline, while it is visible in the experimental spectrum. Vortices are shed alternatively and similarly on each side of the cylinder. The fluctuations induced by this alternate shedding merge equally on the centerline (by mirror-symmetry) so that the resulting streamwise velocity exhibits a characteristic frequency that is twice the frequency of the vortex shedding, i.e. $2 f_{s}$. This feature is well-captured in the simulations, but the peak observed at $f_{s}$ in the experimental spectrum is obviously an artefact. A plausible explanation is that the hot-wire position was not exactly on the centerline but slightly above or below it. In that situation, the recorded fluctuations are always dominated by the vortex shedding on the same side of the cylinder as the hot-wire. This is observed for the spectra in the shear layer at $y / D=0.6$ with a main peak at $f_{s}$. In both positions $y=0$ and $y / D=0.6$, the levels of broadband spectra agree very well between experimental and numerical data up to about $3 \mathrm{kHz}$. Above $3 \mathrm{kHz}$, the numerical simulations over-predict the measurements. The cause of this high-frequency mismatch remains unclear, even if the experimental acquisition chain is preferably questioned (see a comment later). Finally, the dependence on frequency of the spectra is plotted against the characteristic scaling laws $f^{-5 / 3}$ for isotropic turbulence, and $f^{-1}$ for sheared turbulence [42]. As expected, the numerical spectra are found closer to the scaling law $f^{-5 / 3}$ on the centerline, where turbulence is expected to be more isotropic, whereas the scaling law 


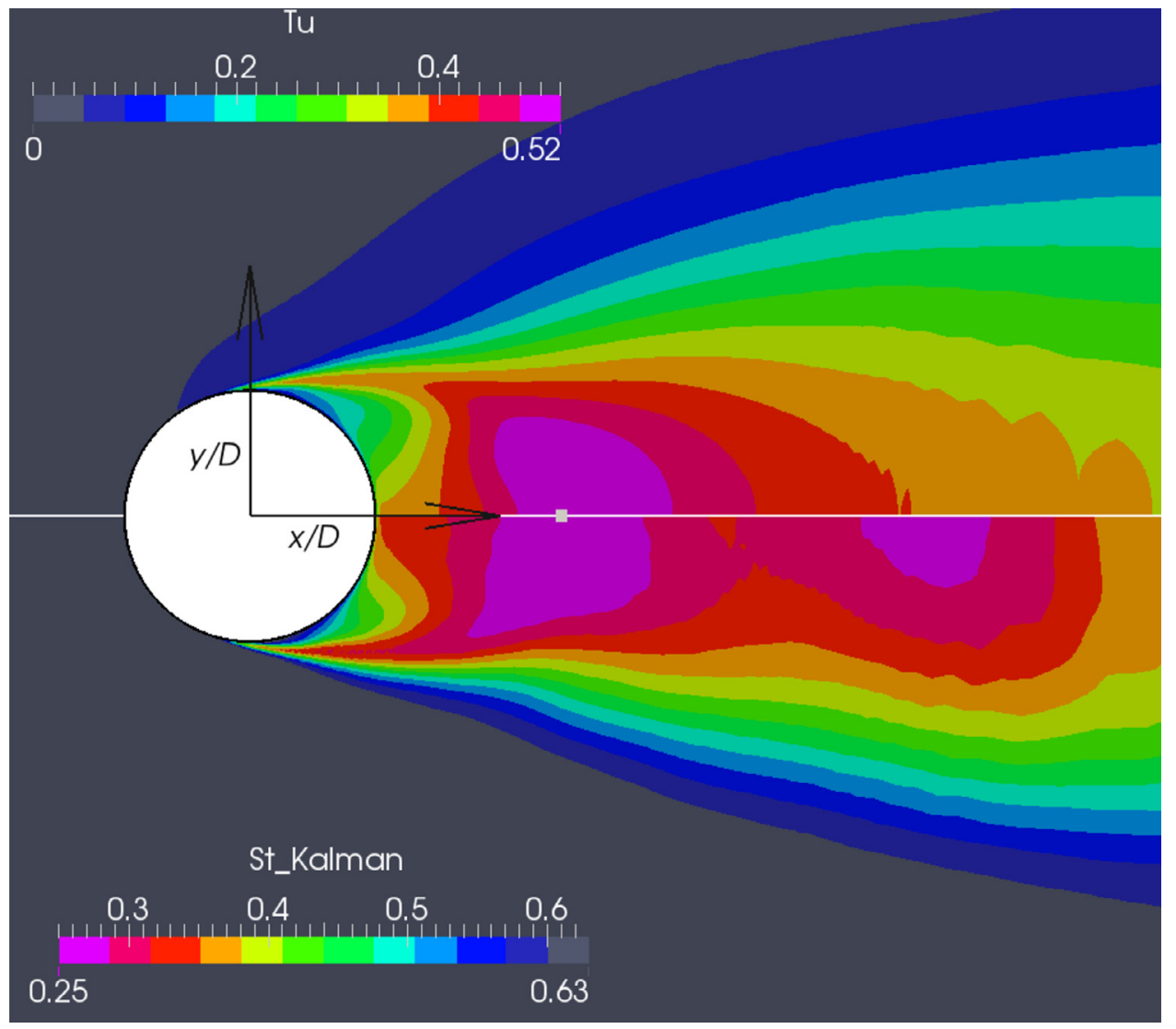

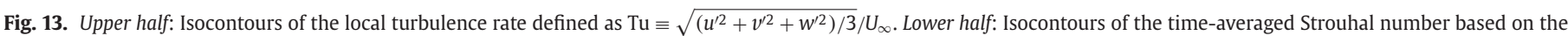

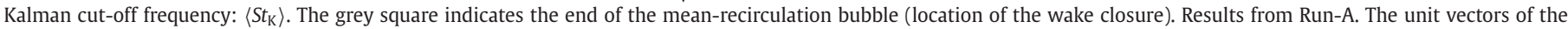
coordinate system $(x / D, y / D)$ are shown.

$f^{-1}$ is more suitable in the shear layer. The three simulations Run-A, $B, C$ yield undistinguishable results.

Since small errors in the simulation of unsteady flows are likely to result in large discrepancies in the acoustic far-field prediction, this latter is also a good tool to assess the quality of unsteady flow computations. It allows us to continue the spectral analysis, beyond the flaws of the hot-wire measurement in the near-wake region. The far field pressure is computed from LES using the FfowcsWilliams and Hawkings acoustic analogy [43]. The present solver, called Turb'AcAn, uses the advanced time approach of Casalino [44] and has been validated on test cases presented in [44]. Integration is performed on the cylinder surface, thus neglecting the quadrupole sources, given the low Mach number considered here $(\sim 0.2)$. The far field spectra at $x / D=15.5$ and $y / D=185$ are compared to in-house measurements [41] in Fig. 10. The numerical spectra have been corrected to correspond to the experimental span length $(30 D)$ by supposing proportionality of the power spectral density, i.e. neglecting correlation for spanwise separations larger than the computational extent. In the experiment, for frequencies below $490 \mathrm{~Hz}$, the cylinder noise does not overpower the background noise, as shown in [41]. Consequently, the spectrum is masked in this region, since the experimental levels are mostly related to background noise. Concerning the simulations, the relatively short time series (67 periods) yield a weaker statistical convergence, which produces wiggles in the spectra.

The general shape of the numerical spectra agrees with the experiment. It is dominated by a very broad peak (more than an octave wide) centered at the shedding frequency. The present result confirms that the shedding frequency is well captured by the simulations. The second and third harmonics can be identified on the experimental spectrum, but only the third harmonic seems to stand out in the simulations. However, the wiggles make the harmonics identification difficult. A particular attention is paid to the broadening of the fundamental peak, associated with turbulence developing in the near wake. It is quite well reproduced by the simulations. Finally, there is a good agreement of the spectrum slopes at high frequencies. This tends to confirm that the discrepancy with the hot-wire results was a measurement artefact. Overall, the agreement between the experimental and numerical spectra in the far field is very satisfactory, for both the fundamental peak and the broadband components.

\section{Behavior of the Kalman filter}

The behavior of the Kalman filter is now examined. In particular, it is important to verify a posteriori that the cut-off frequency of the Kalman filter suitably adapts itself to the local dynamics to extract the large-scale patterns of the flow. In Fig. 11, the left and right images show respectively the $x$-component of the velocity field without and with the smoothing procedure. In the left image, the wake undergoes strong oscillations due to the vortex shedding combined with a variety of turbulent scales. In comparison, the filtered velocity field (represented in the right image) mainly captures the vortex shedding and the turbulent activity has been mostly smoothed out. In the following, a more detailed analysis of the adaptivity of the Kalman filter is presented.

\subsection{Cut-off frequency of the Kalman filter}

As mentioned in Section 2, the optimal gain of our Kalman filter may be interpreted as an adaptive smoothing factor. According to Eq. (3), by analogy with the exponential smoothing, it may be associated formally with a characteristic cut-off frequency $f_{\text {Kalman }}(\mathbf{x}, t)$ 


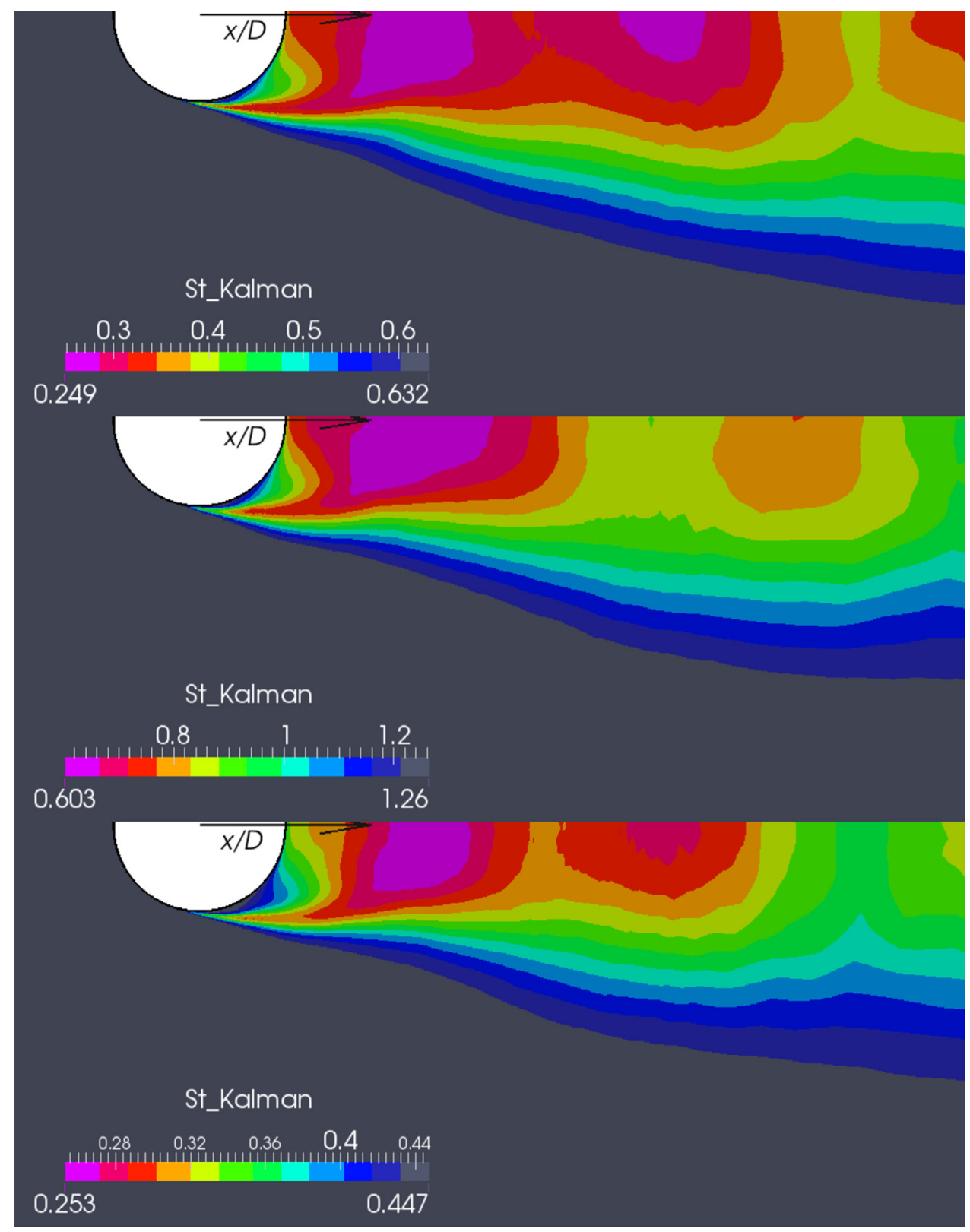

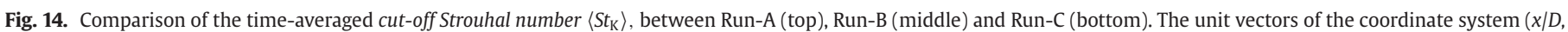
$y / D)$ are shown.

such that

$K(\mathbf{x}, t) \approx 3.628 f_{\text {Kalman }}(\mathbf{x}, t) \cdot \Delta t$

Fig. 12 displays an instantaneous snapshot of the Strouhal number based on the cut-off frequency of the Kalman filter: $S t_{K}(\mathbf{x}, t) \equiv$ $f_{\text {Kalman }}(\mathbf{x}, t) \cdot D / U_{\infty}$ at the same position and time as in Fig. 11, for Run-A. This Strouhal number can be viewed as the normalized cutoff frequency of our Kalman filter. This (normalized) frequency is obviously influenced by the underlying flow dynamics as pointed out by the similarities with Fig. 11. This is better evidenced in Fig. 13, where the time-averaged value $\left\langle S t_{\mathrm{K}}\right\rangle$ is compared with the local turbulence rate of the flow, Tu, for Run-A. The turbulence rate is defined as $\mathrm{Tu} \equiv \sqrt{1 / 3 \cdot\left(u^{\prime 2}+v^{\prime 2}+w^{\prime 2}\right)} / U_{\infty}$, where $u^{\prime}, v^{\prime}$ and $w^{\prime}$ are the root-mean-squared components of the velocity fluctuations. The isocontours of the two fields look very similar, which indicates that (in average) the Kalman cut-off frequency is truly sensitive to the local level of fluctuations of the velocity field. This is a strong assessment of the adaptiveness of our Kalman filter. As expected, the (normalized) cut-off frequency is maximal (so is the Kalman gain) in the external flow region where the fluctuations are weak. In this region, the smoothing is reduced (in average) and the filter is more sensitive to the possible onset of turbulent instabilities. This is a crucial feature around the border of the wake, where intermittent fluctuations are caused by the oscillations of the wake. In the shearlayer-separation zone, where the flow undergoes strong fluctuations with typical frequency $f_{s}$, the (normalized) Kalman frequency suitably adjusts itself to a value slightly above the shedding Strouhal number, thus including the (quasi-)deterministic shedding in the "mean flow". This is physically-sound since the vortex shedding is expected to be part of the mean flow (in the sense of ensemble average). Interestingly, the minimum value of the mean Kalman cut-off frequency is found very close to the wake-closure location, where the mean axial velocity is null and the turbulence rate is roughly maximal. In this region, a lower cut-off frequency enables to efficiently smooth out the turbulent fluctuations. In summary, it is found in the reference simulation Run-A that the cut-off frequency 

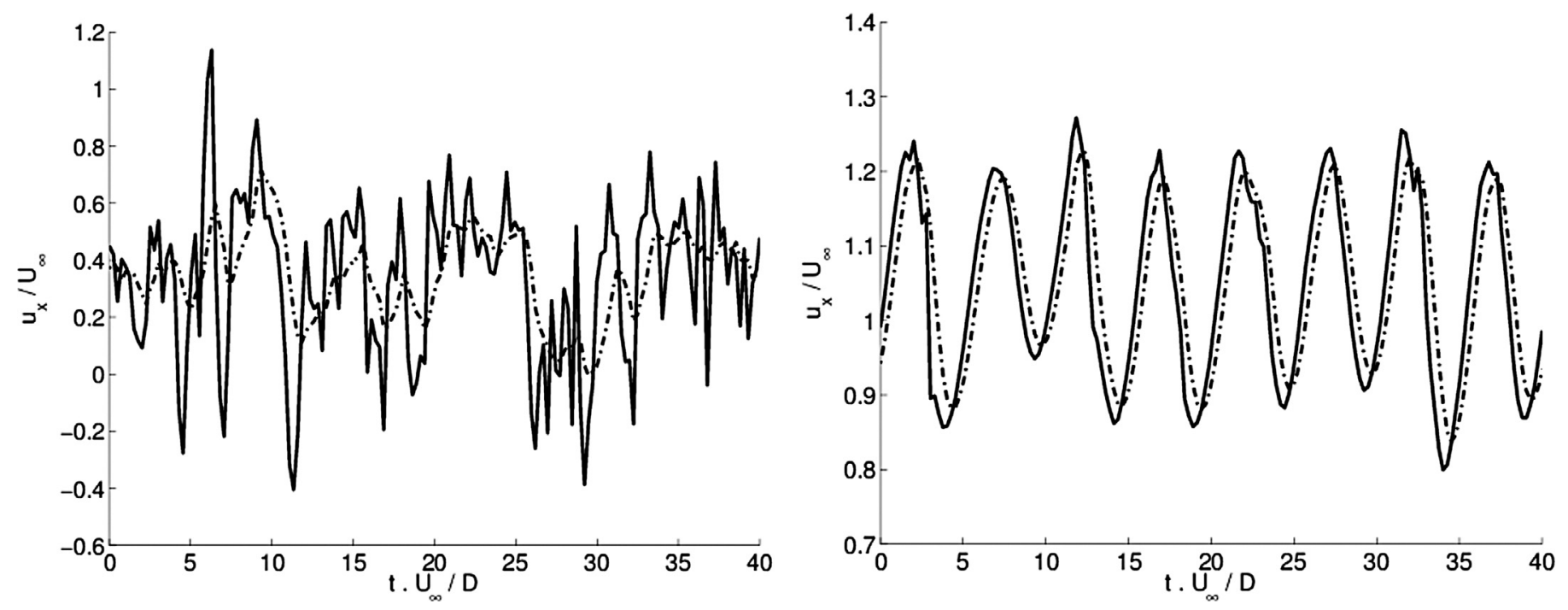

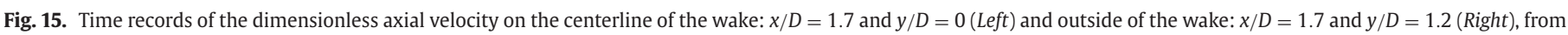
Run-A. -: instantaneous signal, $-\cdot-\cdot-$ : filtered signal.

calculated by our adaptive-Kalman-filter algorithm is physicallysound and suitably reacts to the dynamical structures of the flow.

The time-averaged cut-off Strouhal number $\left\langle S t_{K}\right\rangle$ is compared between Run-A, Run-B and Run-C in Fig. 14. The spatial variations of $\left\langle S t_{K}\right\rangle$ are qualitatively similar for the three runs. However, the $\left\langle S t_{K}\right\rangle$ values are notably increased in Run-B, for which the reference cut-off frequency $f^{*}$ had been intentionally overestimated by a factor 2 (as compared to the reference value used in Run-A). As a consequence, the range of variations of $\left\langle S t_{\mathrm{K}}\right\rangle$ in Run-B is roughly doubled in comparison with Run-A: $0.60<\left\langle S t_{K}\right\rangle<1.26$ in Run-B. The minimum value of $\left\langle S t_{K}\right\rangle$ (reached in the near wake) is notably higher than the shedding Strouhal number $S t=0.2$. Therefore, the turbulent fluctuations in the wake are less-effectively smoothed out, which may explain why Run-B performs less accurately in Section 4. This is visible on the most sensitive quantities: $C p^{\prime}$ in Fig. 5 and the mean wake velocity in Fig. 8. Returning to Fig. 14, the topology of $\left\langle S t_{K}\right\rangle$ along the wake centerline is also affected by the choice of $f^{*}$ : The second minimum observed in Run-A around $x / D=2.5$ is shifted downstream in RunB. Concerning Run-C, the results are very similar to those obtained in Run-A except for a decrease of the maximum value of $\left\langle S t_{\mathrm{K}}\right\rangle$ in the unperturbed flow region outside of the wake. This is the expected behavior since the increase of $\epsilon$ acts on the clipping in the Adapt phase in the algorithm (cf. Table 1).

In summary, it is found that our Kalman filter suitably adapts itself to the dynamical structures of the flow and achieve an efficient filtering of turbulent fluctuations. However, to this end, the reference cut-off frequency $f^{*}$ should be set in reference to the characteristic unsteadiness of the flow. The overestimate of $f^{*}$ in Run-B results in a less-effective smoothing of the turbulent fluctuations and slightly deteriorated results. This should be kept in mind for future applications.

\subsection{Phase delay}

Fig. 15 presents time traces of the instantaneous and filtered axial velocities at two locations: on the centerline of the wake $(x / D=1.7$ and $y / D=0)$ and outside of the wake $(x / D=1.7$ and $y / D=1.2)$, for Run-A. The instantaneous signal on the wake centerline (left plot) is highly turbulent and the smoothing is very satisfactory. In comparison, outside of the wake (right plot), unsteadiness is induced by potential effects associated with the large shed vortices. Therefore, the signal exhibits only low frequencies. In principle, the instantaneous and the smoothed signals should superimpose, but, we observe a phase delay. This delay is due to the fact that the smoothed signal is estimated from past iterations and, thus, cannot follow instantaneously the ongoing slow evolution of the signal. This is a known drawback of all recursive filters. This phase delay can be roughly estimated as

$\tau_{\text {delay }} \approx \frac{\Delta t}{\langle K\rangle} \approx \frac{1}{3.628\left\langle f_{\text {Kalman }}\right\rangle}$

according to [45]. For the signal outside of the wake $(y / D=1.2)$ : $\left\langle S t_{K}\right\rangle$ $\approx 3 \cdot S t \approx 0.6$. One gets $\tau_{\text {delay }} \cdot U_{\infty} / D \approx 0.5$ in good agreement with the observations in Fig. 15 (right plot). This analytical estimate of the delay can be used in relation with Fig. 14, where the time-averaged cut-off Strouhal number is compared for the three computations. Run-A and Run-C display similar values of $\left\langle S t_{K}\right\rangle$ and should therefore present similar delays, except in the unperturbed region where the increased value of $\epsilon$ in Run-C is expected to enhance the delay. In Run-B, higher values of $\left\langle S t_{K}\right\rangle$ are obtained, corresponding to reduced delays. However, as mentioned in the previous section, this reduction comes at the price of a less effective smoothing and slightly degraded results.

In theory, phase delay may be reduced by using a so-called double exponential smoothing. This latter encompasses a second equation that accounts explicitly for the trend of the signal. Along the line of the present work, developing an adaptive double exponential smoothing by use of Kalman filtering is a priori conceivable. However, our simulations indicate that even if phase delay is not satisfactory from a signal-processing viewpoint, it does not seem to have a significant impact on the overall accuracy of the simulation.

\section{Conclusion}

In this work, a Kalman filter adapted to the dynamics of turbulent flows has been introduced and tested. This algorithm is of general interest and efficiently extracts "on the fly" the very-large-scale patterns of a turbulent flow in a numerical simulation. In practice, this information is here profitably used to implement a shear-improved Smagorinsky's subgrid-scale viscosity. The whole methodology aims at addressing the LES of complex unsteady turbulent flows.

The flow past a circular cylinder at high Reynolds numbers is commonly considered as a paradigm of complex flows, involving remarkable features such as thin separating shear layers, transition, and large-scale vortex motion in the wake [28]. In this regard, it has been 
recommended by the Advisory Group for Aerospace Research and Development (AGARD) for the validation of LES solvers. ${ }^{1}$ By treating this severe test case and by comparing our numerical results with various experimental data (in comparable flow configurations), we have demonstrated both the efficiency of our adaptive Kalman filter on its own, and the very good accuracy of the resulting LES. The cutoff frequency of the filter is shown to adapt spatially to the instantaneous flow field. The dependence of the filtering on the calibration frequency $f^{*}$ and the parameter $\epsilon$ has also been evaluated. It appears that only $f^{*}$ has a direct influence on the filtering levels and should therefore be chosen with attention. Nevertheless, in the present configuration, a doubling of this frequency was shown to have a moderate influence on the accuracy of the simulated flow.

Importantly, the whole method is physically-sound. The numerical implementation is efficient in terms of performance and memory usage and the smoothing algorithm remains fully local in space as it applies independently at each grid point. It is therefore particularly adapted for parallelization, and convenient for the formulation of boundary conditions. Nevertheless, further improvements may be achieved, in particular to reduce the phase delay related to the recursivity of the filter. A solution in terms of double exponential smoothing has already been identified and will be investigated in the near future.

\section{Acknowledgments}

E.L. would like to thank $\mathrm{H}$. Touil for valuable discussions on turbulence modeling and CFD. The simulations have been performed by using the local computing facilities at ENS de Lyon (PSMN) supported by the Rhône-Alpes region (CIRA-CPER 2007-2013) and national HPC resources from GENCI-CINES (grant 2014-2a5039).

\section{References}

[1] Ferziger JH, Peric M. Computational methods for fluid dynamics. 3rd Edition. Berlin Heidelberg: Springer-Verlag; 2002.

[2] Hoyas S, Jimenez J. Scaling of the velocity fluctuations in turbulent channels up to $\operatorname{Re}_{\tau}=2003$. Phys Fluids 2006;18(011702).

[3] Sagaut P. Large eddy simulation for incompressible flows: an introduction. 3rd Edition. Berlin Heidelberg: Springer-Verlag; 2006.

[4] Smagorinsky J. General circulation experiments with the primitive equations: I. the basic experiment. Mon Weather Rev 1963:91:99-163.

[5] Lévêque E, Toschi F, Shao L, Bertoglio JP. Shear-improved smagorinsky model for large-eddy simulation of wall-bounded turbulent flows. J Fluid Mech 2007; 570:491-502.

[6] Pope SB. Turbulent flows. Cambridge University Press; 2000

[7] Germano M, Piomelli U, Moin P, Cabot WH. A dynamic subgrid-scale eddyviscosity model. Phys Fluids A 1991;3:1760.

[8] Cahuzac A, Boudet J, Borgnat P, Lévêque E. Smoothing algorithms for mean-flow extraction in large-eddy simulation of complex turbulent flows. Physics of fluids 2010;22:125104.

[9] Toschi F, Lévêque E, Ruiz-Chavarria G. Shear effects in nonhomogeneous turbulence. Phys Rev Lett 2000;85:1436.

[10] Schumann U. Subgrid scale model for finite difference simulations of turbulent flows in plane channels and annulli. J Comput Phys 1975;18:376-404.

[11] Moin P, Kim J. Numerical investigation of turbulent channel flow. J Fluid Mech 1982;118:341-77.

[12] Horiuti K. Comparison of conservative and rotational forms in large eddy simulation of turbulent channel flow. J Comput Phys 1987;71:343-70.
[13] Sullivan PP, McWilliams JC, Moeng CH. A subgrid-scale model for large-eddy simulation of planetary boundary-layer flows. Boundary-Layer Met 1994;71:247-76.

[14] Dubois T, Jauberteau F, Temam R. Dynamic multilevel methods and the numerical simulation of turbulence. Cambridge University Press; 1999.

[15] Hughes TJR, Mazzei L, Jansen KE. Large eddy simulation and the variational multiscale method. Compu Visualiz Sci 2000;3:47.

[16] Berselli LC, Iliescu T, Layton WJ. Mathematics of large eddy simulation of turbulent flows. Springer; 2005.

[17] Ducros F, Comte P, Lesieur M. Large-eddy simulation of transition to turbulence in a boundary layer developing spatially over a flat plate. J Fluid Mech 1996;326:136.

[18] Gardner E. Exponential smoothing: the state of the art. J Forecasting 1985;4:1-48.

[19] Gardner EJ. Exponential smoothing: the state of the art-part ii. Int J Forecasting 2006;22(4):637-66.

[20] Chatelain A. Large eddy simulations of turbulent flows with heat transfer. Thesis, Institut National Polytechnique de Grenoble, France, 2004, https://tel. archives-ouvertes.fr/tel-00006845.

[21] Benarafa Y. RANS/LES coupling applied to high Reynolds number turbulent flows of the nuclear industry, Thesis, Université Pierre et Marie Curie - Paris VI, 2004, https://tel.archives-ouvertes.fr/tel-00011371.

[22] Meneveau C, Lund TS, Cabot WH. A lagrangian dynamic subgrid-scale model of turbulence. J Fluid Mech 1996;319:353-85.

[23] Harvey A. Forecasting, structural time series model and the Kalman filter. Cambridge University Press: 1989.

[24] Brockwell PJ, Davies RA. Time series: theory and methods (Second Edition). Springer series in statistics; 1991.

[25] Boudet J, Caro J, Shao L, Lévêque E. Numerical studies towards practical large-eddy simulation. J Thermal Sci 2007;16(4):328-36.

[26] Boudet J, Monier J-F, Gao F. Implementation of a roughness element to trip transition in large-eddy simulation. J Thermal Sci 2015;24(1).

[27] Williamson CHK. Vortex dynamics in the cylinder wake. Annu Rev Fluid Mech $1996 ; 28: 477-539$

[28] Breuer M. A challenging test case for large eddy simulation: high reynolds number circular cylinder flow. J Heat Fluid Flow 2000;21:648-54.

[29] Kravchenko AG, Moin P. Numerical studies of flow over a circular cylinder at $\operatorname{Re}_{\mathrm{D}}=3900$. Phys Fluids 2000;12(2):403-17.

[30] Szepessy S, Bearman PW. Aspect ratio and end plate effects on vortex shedding from a circular cylinder. J Fluid Mech 1992;234:191-217.

[31] Couregelongue J. On the existence of two families of eddies behind immersed solids. CRAS 1929;189:972-4.

[32] Cantwell B, Coles D. An experimental study of entrainment and transport in the turbulent near wake of a circular cylinder. J Fluid Mech 1983;136:321-74.

[33] Achenbach E. Distribution of local pressure and skin friction around a circular cylinder in cross-flow up to Re $=5 \times 10^{6}$. J Fluid Mech 1968;34:625-39.

[34] Zdravkovich MM. Flow around circular cylinders. Oxford University Press; 2002.

[35] Gerrard JH. An experimental investigation of the oscillating lift and drag of a circular cylinder shedding turbulent vortices. J Fluid Mech 1961;11:244-56.

[36] Norberg C. Fluctuating lift on a circular cylinder: review and new measurments. J Fluids Struct 2003;17:57-96.

[37] Yokuda S, Ramaprian BR. The dynamics of flow around a cylinder at subcritical Reynolds numbers. Phys Fluids A 1990;2(5):784-91.

[38] Anderson EA, Szewczyk AA. Effects of a splitter plate on the near wake of a circular cylinder in 2 and 3-dimensional flow configurations. Exper Fluids 1997;23:16174.

[39] Nishimura H, Taniike Y. Aerodynamic characteristics of fluctuating forces on a circular cylinder. J Wind Eng Ind Aerodyn 2001;89:713-23.

[40] Norberg C. LDV-measurements in the near wake of a circular cylinder. Adv Understanding Bluff Body Wakes Vortex-Inducted Vib 1998:57-96.

[41] Jacob MC, Boudet J, Casalino D, Michard M. A rod-airfoil experiment as a benchmark for broadband noise modelling. Theor Comput Fluid Dyn 2005;19(3):17196.

[42] Tennekes H, Lumley JL. A first course in turbulence. MIT Press; 1972.

[43] Ffowcs-Williams JE, Hawkings DL. Sound generated by turbulence and surfaces in arbitrary motion. Philosophical Trans Royal Soc 1969;A264(1151):321-42.

[44] Casalino D. An advanced time approach for acoustic analogy predictions. J Sound Vib 2003;261(4):583-612.

[45] Papoulis A. Signal analysis. McGraw-Hill; 1984. 